\title{
Explicit HRS-tilting
}

\author{
Behrang Noohi
}

\begin{abstract}
For an abelian category A equipped with a torsion pair, we give an explicit description for the tilted abelian category B introduced in [HaReSm], and also for the categories $\mathrm{Ch}(\mathrm{B})$ and $\mathscr{D}(\mathrm{B})$. We also describe the DG structure on $\mathrm{Ch}(\mathrm{B})$. As a consequence, we find new proofs of certain results of [ibid.]. The main ingredient is the category of decorated complexes.
\end{abstract}

Mathematics Subject Classification (2000). 18E10, 18E40; 18E30, 18G35, 16G10, 16G20, $13 \mathrm{D} 30$.

Keywords. Tilting, stability conditions.

\section{Contents}

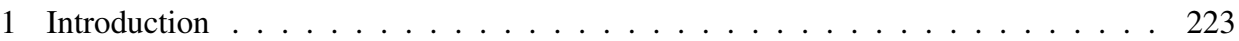

2 A quick review of torsion theories . . . . . . . . . . . . . . . . . 226

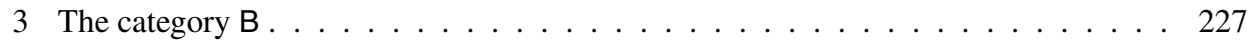

4 Complexes in B and strict morphisms between them . . . . . . . . . . . 233

5 Decorated complexes in A . . . . . . . . . . . . . . . . . 236

6 Complexes in B and the derived category $\mathscr{D}(\mathrm{B}) \ldots \ldots \ldots \ldots . \ldots . \ldots . \ldots 24$

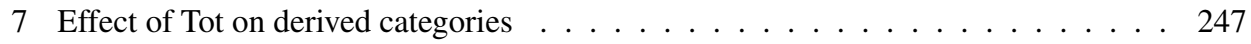

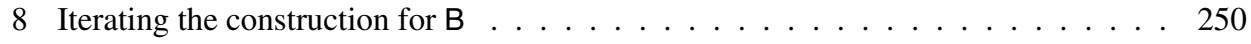

9 The DG structure . . . . . . . . . . . . . . . . . . . . 252

10 The derived DG equivalence between $\mathfrak{C h}^{b}(\mathrm{~B})$ and $\mathfrak{C h}^{b}(\mathrm{~A}, \mathcal{T}, \mathcal{F}) \ldots \ldots . \ldots 25$

References . . . . . . . . . . . . . . . . . . . . . 258

\section{Introduction}

Tilting theory originated from representation theory of (finite dimensional) algebras and their derived categories (see for instance [BeGePa], $[\mathrm{BrBu}]$ for the origins of the theory). It was essentially conceived as a machinery to compare derived categories of various algebras. The theory has developed substantially in the past three decades thanks to the works of various authors such as Auslander, Happel, Keller, Krause, Reiten, Rickard, Ringel, etc. 
Nowadays techniques of tilting theory have found applications in (derived) geometry of varieties, noncommutative geometry, representation theory (of finite groups, algebraic groups, quantum groups, quivers, ...), cluster algebras, and so on.

A precursor to the introduction of the tilting techniques in geometry is the work of Beilinson relating the derived category of coherent sheaves on a projective space to the derived category of a certain finite dimensional noncommutative algebra [Be]. This was further developed by Bondal [Bo] and has now become a standard tool in the study of derived categories of varieties.

Tilting theory is also closely related to Bridgeland's theory of stability conditions $[\mathrm{Br} 2]$. Let us say a few words on this. Given a stability condition $(Z, \mathcal{P})$ on a triangulated category $\mathscr{D}$, the slicing $\mathcal{P}$ gives rise to abelian categories $\mathrm{A}_{\theta}:=\mathscr{P}((\theta, \theta+1])$ inside $\mathscr{D}$. It is easy to see that for $0<\theta \leq 1, \mathrm{~A}_{\theta}$ is obtained by tilting $\mathrm{A}_{0}$ with respect to the torsion pair $\left(\mathcal{T}_{\theta}, \mathcal{F}_{\theta}\right)$, where $\mathcal{F}_{\theta}:=\mathcal{P}((0, \theta])$ and $\mathcal{T}_{\theta}:=\mathcal{P}((\theta, 1])$. This observation has interesting implications in noncommutative geometry. For example, Polishchuk [Po2], [Po1], [PoSch] shows that if we apply this to the derived category $\mathscr{D}(T)$ of coherent sheaves on a complex torus $T$, with the stability condition being the one coming from the Harder-Narasimhan filtration, the tilted abelian category $A_{\theta}$ will be equivalent to the category of coherent sheaves on the noncommutative torus $T_{\theta}$.

For more on the relation between tilting theory and stability conditions on varieties the reader can consult works of Bridgeland and references therein (e.g., [Br1]). An application of tilting theory in noncommutative algebraic geometry appears in [vdB]. Applications to perverse sheaves and representation theory of Lie and quantum groups can be found in various articles by (one or more) of the authors Beilinson, Bezrukavnikov, Mirkovic, etc.

One of the main tools in the works mentioned above is the construction of the 'tilting' of an abelian category with respect to a torsion pair [HaReSm]. In this article the authors associate to an abelian category $A$ equipped with a torsion pair $(\mathcal{T}, \mathcal{F})$ a new abelian category $B$ (which is in turn equipped with its own torsion pair $\left(\mathcal{T}^{\prime}, \mathscr{F}^{\prime}\right)$ ). This is the 'HRS-tilting' of A.

The construction of $B$ in $[\mathrm{HaReSm}]$ is indirect and is carried out by taking the heart of a certain $t$-structure (associated to the torsion pair) on the derived category of A. In this article we give an alternative construction for $B$ that is more explicit and reveals more of the structure of $B$, as we explain shortly. We expect this new description to be suitable for geometric applications, as the explicit nature of B lends itself well to geometric manipulations (say when working with bundles over a variety). It could very well give a new insight to the category theoretic properties (say, existence of generators, chain conditions, limits and colomits, etc.) of B as well.

The main input in this work is an alternative description of morphisms in the derived category $\mathscr{D}(\mathrm{A})$ between complexes concentrated in degrees $[-1,0]$; see $([\mathrm{No}]$, Section 9) and Section 3.1. We exploit this to give an explicit description of the category $\mathrm{Ch}(\mathrm{B})$ of chain complexes in B, its DG structure (Sections 9 and 10, especially, 
Theorem 10.9), and its derived category (Theorem 7.3). This is achieved via what we call a decorated complex, which might be a notion of independent interest; see Section 5. The correspondence between the homological algebra of B and that of decorated complexes in $A$ is established via a functor Tot which should be thought of as a "twisted" total complexes functor.

Although it is not the main purpose of the paper, we also show how our approach leads to new proofs for some of the main results of Happel-Reiten-Smal $\varnothing$; see Theorem 7.6 and Theorem 8.2.

Outline of the main results. Let $(\mathcal{T}, \mathcal{F})$ be a torsion pair on an abelian category $A$. We begin by observing that, by results of [No], the abelian category obtained by performing HRS-tilting on this torsion pair is equivalent to the following category:

- $\mathrm{Ob}(\mathrm{B})=\left\{\mathbb{X}=\left[X^{-1} \stackrel{d}{\rightarrow} X^{0}\right] \mid \operatorname{ker} d \in \mathcal{F}\right.$, coker $\left.d \in \mathcal{T}\right\}$.

- $\operatorname{Hom}_{\mathrm{B}}(\mathbb{X}, \mathbb{Y})=$ isomorphism classes of commutative diagrams

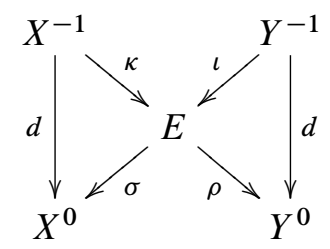

such that the diagonal maps compose to zero and the NE-SW sequence is short exact.

We use this description to get explicit information about B. For instance, the kernel and cokernel of a 'butterfly' diagram $P$ as above are given by

$$
\begin{aligned}
\operatorname{ker} P & :=\left[X^{-1} \stackrel{\kappa}{\rightarrow} A\right], \\
\operatorname{coker} P & :=\left[E / A \stackrel{\rho}{\rightarrow} Y^{0}\right] .
\end{aligned}
$$

Here $A$ is the (unique) subobject of $E$ sitting between $\operatorname{im} \kappa$ and ker $\rho$ such that $A / \operatorname{im}(\kappa) \in \mathcal{T}$ and $\operatorname{ker}(\rho) / A \in \mathcal{F}$. Using this we find a description of complexes in B; see Section 6.2.

We then exploit these results to give a description of the derived category of $B$ in terms of decorated complexes (Section 5). A decorated complex in A consists of a complex $E^{\bullet}$ in $A$, together with a collection of subobjects $M^{n} \subseteq C^{n}$ for every $n$. The differentials of $E^{\bullet}$ are not required to respect the subobjects. A morphism $\left(E^{\bullet}, M^{\bullet}\right) \rightarrow\left(F^{\bullet}, N^{\bullet}\right)$ of decorated complexes is, by definition, a chain map $f: E^{\bullet} \rightarrow F^{\bullet}$ which respects the subobjects. We say that such a morphism is a quasi-isomorphism if $f$ is so.

A decorated complex $\left(E^{\bullet}, M^{\bullet}\right)$ is said to be compatible with a torsion pair $(\mathcal{T}, \mathcal{F})$ if 
- $M^{n} \cap \partial^{-1} M^{n+1} \in \mathcal{F}$ and $E^{n} /\left(M^{n}+\partial M^{n-1}\right) \in \mathcal{T}$ for every $n$.

The decorated complexes whose decoration is compatible with the torsion pair form a full subcategory of $\operatorname{Dec}(A)$ which we denote by $\operatorname{Ch}(A, \mathcal{T}, \mathcal{F})$. We have the following description of the derived category $\mathscr{D}(\mathrm{B})$ of $\mathrm{B}$ (see Corollary 6.2).

Theorem 1.1. There is a natural equivalence of triangulated categories

$$
\operatorname{Ch}(\mathrm{A}, \mathcal{T}, \mathcal{F}) / \mathrm{qis} \cong \mathscr{D}(\mathrm{B}) .
$$

The same this is true for bounded (above, below, both-sided) derived categories.

The category $\operatorname{Dec}(A)$ of decorated complexes in $A$ behaves very much like the category $\mathrm{Ch}(\mathrm{A})$ of chain complexes in that we can define decorated cylinders, cones, homotopies, and so on. In other words, we can do homological algebra in $\operatorname{Dec}(A)$. In particular, we can talk about the homotopy and the derived categories of $\operatorname{Dec}(A)$, and these are both triangulated categories.

In the case where $\mathrm{A}$ is the category of $K$-modules for a ring $K, \operatorname{Dec}(K)$ is a closed monoidal category. More generally, if A is $K$-linear, then $\operatorname{Dec}(\mathrm{A})$ is enriched over $\operatorname{Dec}(K)$. This way $\operatorname{Ch}(\mathrm{A}, \mathcal{T}, \mathcal{F})$ inherits a DG structure from $\operatorname{Dec}(\mathrm{A})$, which we denote by $\mathfrak{C h}(A, \mathcal{T}, \mathcal{F})$. Also, $\mathrm{Ch}(\mathrm{B})$ has a DG structure, which we denote by $\left(\mathfrak{C h} \mathfrak{h}^{*}(\mathrm{~B})\right.$.

The above theorem can now be enhanced to a derived equivalence of derived categories; see Theorem 10.9.

Theorem 1.2. Assume that $\mathrm{A}$ has either enough injectives or enough projectives. Assume further that B has enough injectives (respectively, enough projectives). Let $*=+, b$ (respectively, $*=-, b)$. Then we have a derived equivalence

$$
(\mathfrak{C h})^{*}(A, \mathcal{T}, \mathcal{F}) \cong(\mathfrak{C h})^{*}(B)
$$

of DG categories.

Finally, let us remark that, in view of the above results, the functor $D(\mathrm{~B}) \rightarrow D(\mathrm{~A})$ (and the DG functor $\mathfrak{C h}(B) \rightarrow \mathfrak{C h}(A)$ ) studied in $[\mathrm{HaReSm}]$ is nothing but the forgetful functor

$$
\left(E^{\bullet}, M^{\bullet}\right) \mapsto E^{\bullet}
$$

that forgets the decoration. In the case where the torsion pair is tilting or cotilting this is known to be an (derived) equivalence; see Theorem 7.6.

\section{A quick review of torsion theories}

Let $A$ be an abelian category. A torsion theory in $A$ is a pair $(\mathcal{T}, \mathcal{F})$ of full additive subcategories of A such that: 
- for every $T \in \mathcal{T}$ and $F \in \mathcal{F}$, we have $\operatorname{Hom}(T, F)=0$;

- for every $A \in \mathrm{A}$, there is a (necessarily unique) exact sequence

$$
0 \rightarrow T \rightarrow A \rightarrow F \rightarrow 0, \quad T \in \mathcal{T}, F \in \mathscr{F} .
$$

The following facts are well-known and easy to prove.

Lemma 2.1. For a torsion theory $(\mathcal{T}, \mathcal{F})$ we have $\mathcal{T}^{\perp}=\mathscr{F}$ and ${ }^{\perp} \mathcal{F}=\mathcal{T}$, that is,

$$
\begin{aligned}
& \mathscr{F}=\{F \in \mathrm{A} \mid \operatorname{Hom}(T, F)=0 \text { for all } T \in \mathcal{T}\}, \\
& \mathcal{T}=\{T \in \mathrm{A} \mid \operatorname{Hom}(T, F)=0 \text { for all } F \in \mathcal{F}\} .
\end{aligned}
$$

Lemma 2.2. If $X \rightarrow Y$ is a monomorphism and $Y$ is in $\mathcal{F}$, then $X$ is in $\mathcal{F}$. If $Y \rightarrow Z$ is an epimorphism and $Y$ is in $\mathcal{T}$, then $Z$ is in $\mathcal{T}$.

Observe that it is not true in general that a subobject of an object $Y \in \mathcal{T}$ is in $\mathcal{T}$. Similarly, it is not true in general that a quotient of an object $Y \in \mathscr{F}$ is in $\mathscr{F}$.

Lemma 2.3. Consider the exact sequence

$$
0 \rightarrow X \rightarrow Y \rightarrow Z \rightarrow 0
$$

in $\mathrm{A}$. If $X$ and $Z$ are both in $\mathcal{T}$ (respectively, in $\mathcal{F}$ ), then so is $Y$.

\section{The category $B$}

Let $\mathrm{A}$ be an abelian category and $(\mathcal{T}, \mathcal{F})$ a torsion pair in $\mathrm{A}$. To this data we associate a new abelian category $B$ and a torsion pair $\left(\mathcal{T}^{\prime}, \widetilde{F}^{\prime}\right)$. By results of [No], Section 9, this category is naturally equivalent to the one defined in [HaReSm].

3.1. Definition of B. The category $B$ is defined as follows:

- $\mathrm{Ob}(\mathrm{B})=\left\{\mathbb{X}=\left[X^{-1} \stackrel{d}{\rightarrow} X^{0}\right] \mid \operatorname{ker} d \in \mathcal{F}\right.$, coker $\left.d \in \mathcal{T}\right\}$. We will usually $\operatorname{drop} d$ from the notation.

- $\operatorname{Hom}_{\mathrm{B}}(\mathbb{X}, Y)=$ isomorphism classes of commutative diagrams

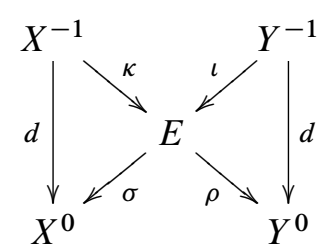

such that the diagonal maps compose to zero and the NE-SW sequence is short exact. 
Remark 3.1. It follows from the axioms of a torsion pair that, given objects $\mathbb{X}$ and $Y$ in $\mathrm{B}$ and two diagrams $E$ and $E^{\prime}$ as above, there exists at most one isomorphism between $E \rightarrow E^{\prime}$ commuting with all the four arrows of the two diagrams. Therefore, by passing to isomorphism classes of such diagrams we do not loose any information.

A morphism that comes from an actual morphism of complexes $f: \mathbb{X} \rightarrow Y$ in $\mathrm{Ch}(\mathrm{A})$ corresponds to the diagram

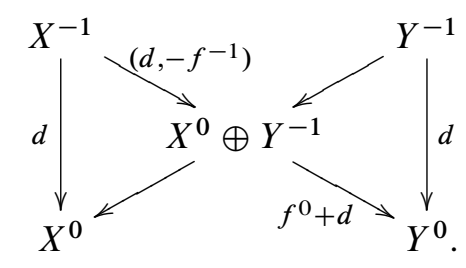

For simplicity, we denote such morphisms in the usual way

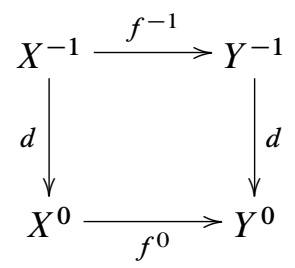

and call them strict morphisms. Equivalently, a strict morphism in B is one for which the NE-SW sequence splits.

Lemma 3.2. If $\mathbb{X}$ is such that $X^{0}$ is projective, then every morphism coming out of $\mathbb{X}$ is strict. If $Y$ is such that $Y^{-1}$ is injective, then every morphism to $Y$ is strict.

Proof. Trivial.

3.2. Composition of morphisms. Given two morphisms
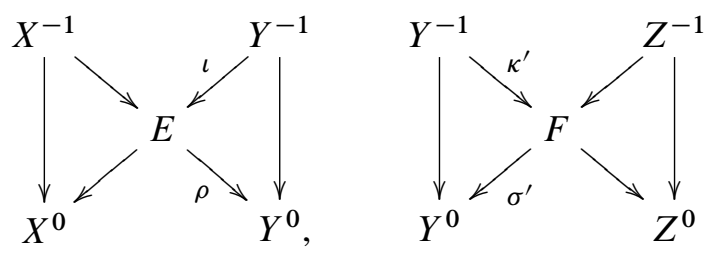
in $\mathrm{B}$, we define their composition to be

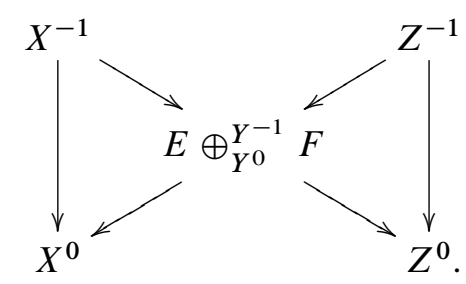

Here $E \oplus_{Y^{0}}^{Y^{-1}} F$ is the quotient of the object $L$ consisting of pairs $(x, y) \in E \times F$ such that $\rho(x)=\sigma^{\prime}(y) \in Y^{0}$, modulo the subobject $I=\left\{\left(\iota(\beta), \kappa^{\prime}(\beta)\right) \in E \times F \mid\right.$ $\left.\beta \in Y^{-1}\right\}$. More precisely, let $E \oplus_{Y^{0}} F$ be the fiber product of $E$ and $F$ over $Y^{0}$. Then

$$
E \oplus_{Y^{0}}^{Y^{-1}} F:=\operatorname{coker}\left(Y^{-1} \stackrel{\left(\iota, \kappa^{\prime}\right)}{\longrightarrow} E \oplus_{Y^{0}} F\right) .
$$

In the case where one of the morphisms is strict, the composition takes a simpler form. When the first morphisms is strict, say

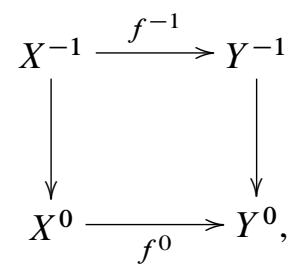

then the composition is

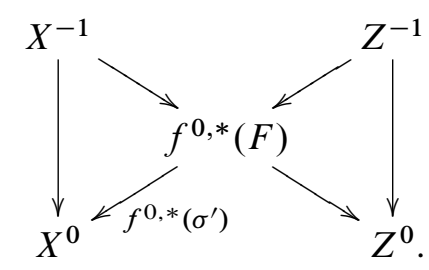

Here $f^{0, *}(F)$ stands for the pull back of the extension $F$ along $f^{0}: X^{0} \rightarrow Y^{0}$. More precisely, $f^{0, *}(F)=X^{0} \oplus_{Y^{0}} F$ is the fiber product.

When the second morphisms is strict, say

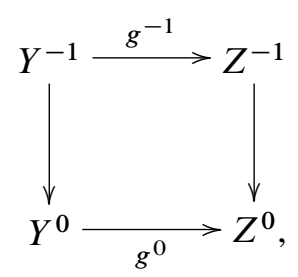


then the composition is

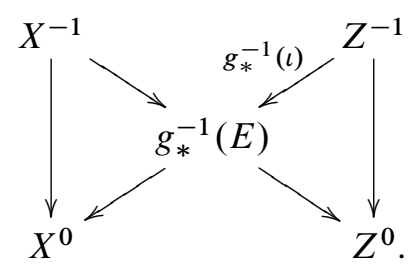

Here $g_{*}^{-1}(E)$ stands for the push forward of the extension $E$ along $g^{-1}: Y^{-1} \rightarrow Z^{-1}$. More precisely, $g_{*}^{-1}(E)=E \oplus^{Y^{-1}} Z^{-1}$ is the push-out.

3.3. Addition of morphisms. Given two elements $P, P^{\prime} \in \operatorname{Hom}(\mathbb{X}, \mathbb{Y})$,
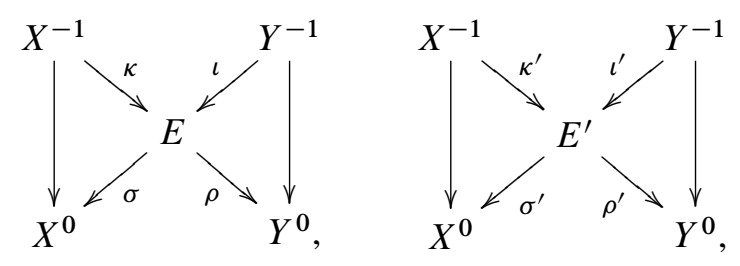

we define $P+P^{\prime} \in \operatorname{Hom}(\mathbb{X}, Y)$ to be

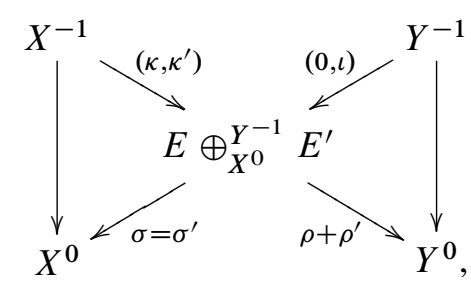

where $E \oplus_{X^{0}}^{Y^{-1}} E^{\prime}$ is defined as in Section 3.2, with the difference that now we mod out $E \oplus_{X^{0}} E^{\prime}$ by the antidiagonal image of $Y^{-1}$ instead of the diagonal image. The map in the bottom-left corner, denoted by $\sigma=\sigma^{\prime}$, sends $(a, b)$ to $\sigma(a)$ (which, by definition, is equal to $\sigma^{\prime}(b)$ ).

We define $-P$ by

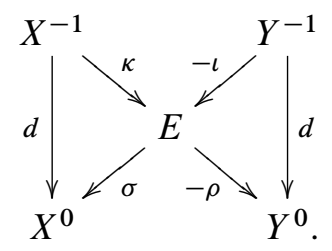

When $\mathrm{A}$ is $R$-linear for some commutative ring $R$, then $\mathrm{B}$ is also naturally $R$ linear. For $r \in R$ and $P$ as above, $r P$ is equal to the composition of $P$ and the strict morphism $r \cdot-: Y \rightarrow Y$; examine the end of Section 3.2 to see what this exactly means. In the case where $r$ is a unit, $r P$ is represented by $\left(E, \kappa, r^{-1} \iota, \sigma, r \rho\right)$. 
3.4. Kernels, cokernels. Consider $P \in \operatorname{Hom}(\mathbb{X}, \mathcal{Y})$ given by

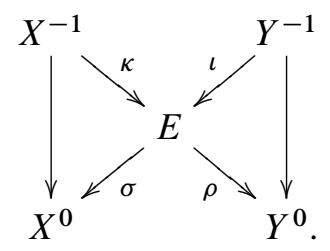

The cone of $P$, where now we consider $P$ as a morphism in the derived category $\mathscr{D}(\mathrm{A})$, has a natural model, namely, the NW-SE complex

$$
C(P):=X^{-1} \stackrel{\kappa}{\rightarrow} E \stackrel{\rho}{\rightarrow} Y^{0},
$$

in which $Y^{0}$ is sitting in degree 0 . The corresponding triangle

$$
\mathbb{X} \rightarrow \mathrm{Y} \rightarrow C(P) \rightarrow \mathbb{X}[1]
$$

is defined in the obvious way.

From this we get the following descriptions of the kernel and cokernel of $P$ in $\mathrm{B}$.

Kernel. Let $A=q^{-1}(T)$, where $T \in \mathcal{T}$ is the torsion part of $H^{-1}(C(P))$ and $q$ : $\operatorname{ker} \rho \rightarrow H^{-1}(C(P))$ is the quotient map. Then the kernel of $P$ is

$$
\operatorname{ker} P:=\left[X^{-1} \stackrel{\kappa}{\rightarrow} A\right] \text {. }
$$

The map ker $P \rightarrow \mathbb{X}$ is given by $\left(\operatorname{id}_{X^{-1}},\left.\sigma\right|_{A}\right)$. We have $H^{-1}(\operatorname{ker}(P))=H^{-2}(C(P))$ and $H^{0}(\operatorname{ker}(P))=T$.

Cokernel. The cokernel of $P$ is

$$
\operatorname{coker} P:=\left[E / A \stackrel{\rho}{\rightarrow} Y^{0}\right]
$$

The map $Y \rightarrow$ coker $P$ is given by $\left(\iota, \operatorname{id}_{Y^{0}}\right)$. We have $H^{-1}(\operatorname{coker}(P))=F$, the free part of $H^{-1}(C(P))$, and $H^{0}(\operatorname{coker}(P))=H^{0}(C(P))$

Corollary 3.3. A morphism $P$ as above is a monomorphism if and only if $\kappa$ is a monomorphism and $H^{-1}(C(P)) \in \mathcal{F}$. The morphism $P$ is an epimorphism if and only if $\rho$ is an epimorphism and $H^{-1}(C(P)) \in \mathcal{T}$.

Corollary 3.4. A morphism $P$ as above is an isomorphism if and only if the NW-SE sequence $C(P)$ is short exact. In this case, the inverse of $P$ is obtained by flipping the diagram with respect to the vertical axis. 
Corollary 3.5. Let $f: \mathbb{X} \rightarrow \mathbb{Y}$ be a strict morphism that is an equivalence. Then the inverse $f^{-1}: \mathbb{Y} \rightarrow \mathbb{X}$ corresponds to the diagram

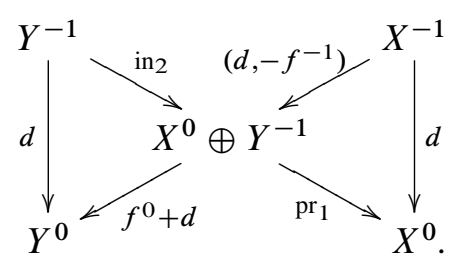

Proof. Use the discussion of Section 3.1 to find the diagram corresponding to $f$. Then flip the diagram.

The short exact sequence

$$
0 \rightarrow \operatorname{ker} P[1] \rightarrow C(P) \rightarrow \operatorname{coker}(P) \rightarrow 0
$$

of complexes gives rise to the exact sequence

$$
0 \rightarrow H^{0}(\operatorname{ker} P) \rightarrow H^{-1}(C(P)) \rightarrow H^{-1}(\operatorname{coker} P) \rightarrow 0
$$

of cohomologies.

We also have the following result.

Proposition 3.6. There is a long exact sequence

$$
\begin{aligned}
0 \rightarrow H^{-2}(C(P)) & \rightarrow H^{-1}(\mathbb{X}) \rightarrow H^{-1}(\mathbb{Y}) \rightarrow \\
& \rightarrow H^{-1}(C(P)) \rightarrow H^{0}(\mathbb{X}) \rightarrow H^{0}(\mathcal{Y}) \rightarrow H^{0}(C(P)) \rightarrow 0 .
\end{aligned}
$$

Proof. This is the exact sequence for the exact triangle $\mathbb{X} \rightarrow Y \rightarrow C(P) \rightarrow \mathbb{X}[1]$.

3.5. The epi-mono factorization. Notation being as in Section 3.4, it is easy to see that the cokernel of the map $\operatorname{ker} P \rightarrow \mathbb{X}$ is the complex

$$
\operatorname{coim} P:=\left[A \stackrel{\sigma}{\rightarrow} X^{0}\right],
$$

and the kernel of $Y \rightarrow \operatorname{coker} P$ is the complex

$$
\text { im } P:=\left[Y^{-1} \stackrel{\text { prol }}{\longrightarrow} E / A\right] .
$$

There is a canonical isomorphism coim $P \rightarrow \operatorname{im} P$ given by

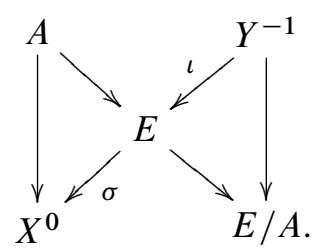


(See Corollary 3.4.) So the epi-mono factorization of $P$ looks like

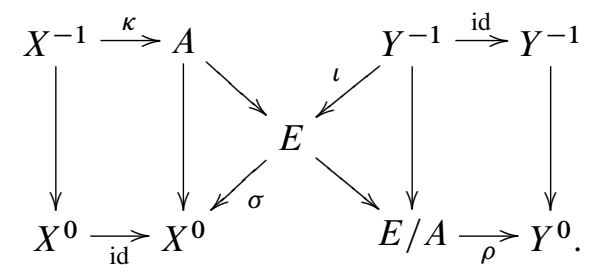

\section{Complexes in B and strict morphisms between them}

In this section we prepare ourselves for the first main result of this article that will appear in Section 6.1. One of the major players here is the category $\mathrm{Ch}^{\text {st }}(\mathrm{B})$ defined below.

Let $\mathrm{Ch}^{\text {st }}(\mathrm{B}) \subset \mathrm{Ch}(\mathrm{B})$ be the category whose objects are complexes

$$
X=\cdots \rightarrow{ }^{n-1} \mathbb{X} \rightarrow{ }^{n} \mathbb{X} \rightarrow{ }^{n+1} \mathbb{X} \rightarrow \cdots
$$

of objects in B, and whose morphisms are strict morphisms of complexes, that is, morphisms $\boldsymbol{X} \rightarrow \boldsymbol{Y}$ such that for every $n$ the morphism ${ }^{n} \mathbb{X} \rightarrow{ }^{n} Y$ is strict; see Section 3.1.

We define two classes of morphisms $\delta_{\text {sis }} \subset \delta_{\text {qis }}$ in $\mathrm{Ch}^{\text {st }}(\mathrm{B})$. The class $\gamma_{\text {sis }}$ consists of morphisms $s: \boldsymbol{X} \rightarrow \boldsymbol{Y}$ that become isomorphisms in $\mathrm{Ch}(\mathrm{B})$; note that $s^{-1}$ may no longer be strict, so $s$ is not necessarily an isomorphism in $\mathrm{Ch}^{\text {st }}(\mathrm{B})$. The class $\boldsymbol{S}_{\mathrm{qis}}$ consists of all quasi-isomorphisms in $\mathrm{Ch}^{\text {st }}(\mathrm{B})$.

The class $\delta_{\text {sis }}$ is indeed a localizing class. This follows from Lemma 4.2 below.

Lemma 4.1. Let $P: \mathbb{X} \rightarrow Y$ be a morphism in $\mathrm{B}$. Then there is a functorial commutative diagram

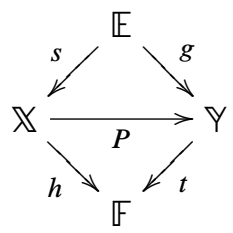

in $\mathrm{B}$ such that $s, t, g$ and $h$ are strict (Section 3.1) and $s$ and t are isomorphisms (note that $s^{-1}$ and $t^{-1}$ are no longer strict). 
Proof. First we prove the existence of $s$ and $g$. Consider the diagram for $P$

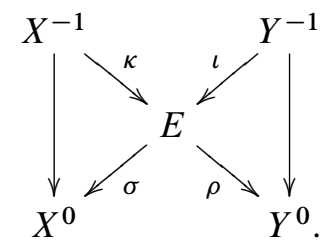

We define

$$
\mathbb{E}:=\left[X^{-1} \oplus Y^{-1} \stackrel{\kappa+\iota}{\longrightarrow} E\right] .
$$

The strict map $s: \mathbb{E} \rightarrow \mathbb{X}$ is given by $\left(\operatorname{pr}_{1}, \sigma\right)$ and is easily seen to be an isomorphism. The map $g: \mathbb{E} \rightarrow \mathcal{Y}$ is defined by $\left(\mathrm{pr}_{2}, \rho\right)$.

The construction of $t$ and $h$ is similar. We take

$$
\mathbb{F}:=\left[E \stackrel{(\sigma, \rho)}{\longrightarrow} X^{0} \oplus Y^{0}\right]
$$

The strict map $t: Y \rightarrow \mathbb{F}$ is given by $\left(\iota, \mathrm{in}_{2}\right)$ and is easily seen to be an isomorphism. The map $h: \mathbb{X} \rightarrow \mathbb{F}$ is defined by $\left(\kappa, \mathrm{in}_{1}\right)$.

Let us prove the functoriality of $\mathbb{E}$. Consider the commutative diagram

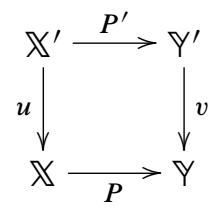

and let $\mathbb{E}$ and $\mathbb{E}^{\prime}$ be constructed as above. Let $w: \mathbb{E} \rightarrow \mathbb{E}^{\prime}$ be $s^{-1} \circ u \circ s^{\prime}$. It is easy to see that $w$ commutes with both $s$ maps and the $g$ maps; in fact $w$ is uniquely determined by this property. (Observe that we did not require $w$ to be strict.)

The functoriality of $\mathbb{F}$ is proved in a similar way.

Lemma 4.2. Let $f: X \rightarrow Y$ be a morphism in $\mathrm{Ch}(\mathrm{B})$. Then there is a commutative diagram

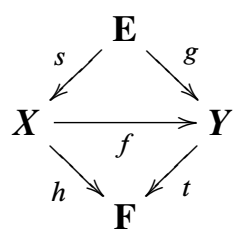

in $\mathrm{Ch}(\mathrm{B})$ such that $s, t, g$ and $h$ are in $\mathrm{Ch}^{\mathrm{st}}(\mathrm{B})$ and $s$ and $t$ are in $8_{\text {sis. }}$.

Proof. This follows immediately from Lemma 4.1. 
Proposition 4.3. The inclusion $\mathrm{Ch}^{\mathrm{st}}(\mathrm{B}) \hookrightarrow \mathrm{Ch}(\mathrm{B})$ induces the following equivalences of categories:

$$
\begin{aligned}
& \wp_{\text {sis }}^{-1} \mathrm{Ch}^{\text {st }}(\mathrm{B}) \stackrel{\sim}{\longrightarrow} \mathrm{Ch}(\mathrm{B}), \\
& \mathcal{f}_{\mathrm{qis}}^{-1} \mathrm{Ch}^{\mathrm{st}}(\mathrm{B}) \stackrel{\sim}{\longrightarrow} \mathcal{D}(\mathrm{B}) .
\end{aligned}
$$

Proof. The first equivalence follows immediately from Lemma 4.2. The second equivalence follows from the first equivalence.

We will need the following lemma in Section 8.

Lemma 4.4. Let $a<b$ be two integers. Then the full subcategory ${ }^{[a, b]} \mathrm{Ch}^{\text {st }}(\mathrm{B})$ of $\mathrm{Ch}^{\text {st }}(\mathrm{B})$ consisting of complexes concentrated in degrees lying in the interval $[a, b]$ is localizing with respect to both $\boldsymbol{\gamma}_{\mathrm{sis}}$ and $\boldsymbol{\wp}_{\mathrm{qis}}$. That is, we have fully faithful functors:

$$
\begin{aligned}
& P: \delta_{\text {sis }}^{-1[a, b]} \mathrm{Ch}^{\mathrm{st}}(\mathrm{B}) \rightarrow \delta_{\text {sis }}^{-1} \mathrm{Ch}^{\mathrm{st}}(\mathrm{B}), \\
& Q: \delta_{\mathrm{qis}}^{-1[a, b]} \mathrm{Ch}^{\mathrm{st}}(\mathrm{B}) \rightarrow \delta_{\mathrm{qis}}^{-1} \mathrm{Ch}^{\mathrm{st}}(\mathrm{B}) .
\end{aligned}
$$

(Here, by abuse of notation, we denote $\delta_{\text {sis }}^{-1} \cap{ }^{[a, b]} \mathrm{Ch}^{\text {st }}(\mathrm{B})$ and $\delta_{\mathrm{qis}}^{-1} \cap^{[a, b]} \mathrm{Ch}^{\text {st }}(\mathrm{B})$ also by $\boldsymbol{\delta}_{\mathrm{sis}}$ and $\boldsymbol{\delta}_{\mathrm{qis}}$.) The same thing is true if we take the full subcategory of complexes $\boldsymbol{X}$ in ${ }^{[a, b]} \mathrm{Ch}^{\mathrm{st}} \mathrm{B}$ such that $H^{a}(\boldsymbol{X}) \in \mathcal{F}^{\prime}$ and $H^{b}(\boldsymbol{X}) \in \mathcal{T}^{\prime}$.

Proof. The case of $\boldsymbol{\delta}_{\text {sis }}$ is obvious. Let us prove the case of $\boldsymbol{\delta}_{\text {qis }}$. Let K(B) and ${ }^{[a, b]} \mathrm{K}(\mathrm{B})$ be the homotopy categories of $\mathrm{Ch}^{\text {st }}(\mathrm{B})$ and ${ }^{[a, b]} \mathrm{Ch}^{\text {st }}(\mathrm{B})$. It is enough to prove the statement for the full subcategory ${ }^{[a, b]} \mathrm{K}(\mathrm{B}) \subseteq \mathrm{K}(\mathrm{B})$. We will denote the class of quasi-isomorphisms in $\mathrm{K}(\mathrm{B})$ by $\delta$. Note that this is a localizing class. By abuse of notation, we denote $\delta \cap{ }^{[a, b]} \mathrm{K}(\mathrm{B})$ also by $\delta$.

Let $\tau_{\geq a}, \tau_{\leq b}: \mathrm{Ch}^{\text {st }}(\mathrm{B}) \rightarrow \mathrm{Ch}^{\text {st }}(\mathrm{B})$ be the usual truncation functors that we know from the theory of $t$-structures, and let $\tau_{[a, b]}=\tau_{\geq a} \circ \tau_{\leq b}: \mathrm{Ch}^{\text {st }}(\mathrm{B}) \rightarrow{ }^{[a, b]} \mathrm{Ch}^{\text {st }}(\mathrm{B})$. We use the same notation $\tau_{[a, b]}$ for the induced functor on the homotopy categories as well as the localized categories. Let $\boldsymbol{X}, \boldsymbol{Y} \in{ }^{[a, b]} \mathrm{K}(\mathrm{B})$. We have to show that the map

$$
\alpha: \operatorname{Hom}_{\mathcal{S}^{-1}([a, b] \mathrm{K}(\mathrm{B}))}(\boldsymbol{X}, \boldsymbol{Y}) \rightarrow \operatorname{Hom}_{\mathcal{S}^{-1} \mathrm{~K}(\mathrm{~B})}(Q \boldsymbol{X}, Q \boldsymbol{Y})
$$

induced by $Q$ is an isomorphism. Let

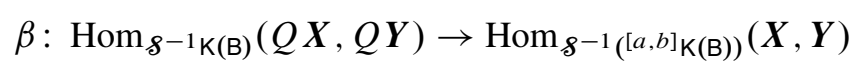

be the map induced by $\tau_{[a, b]}$. We show that $\alpha$ and $\beta$ are inverse to each other. It is clear that $\beta \circ \alpha=1$. To prove $\alpha \circ \beta=1$, let $\tilde{f} \in \operatorname{Hom}_{\mathcal{S}^{-1} \mathrm{~K}(\mathrm{~B})}(Q \boldsymbol{X}, Q \boldsymbol{Y})$ be given by the roof

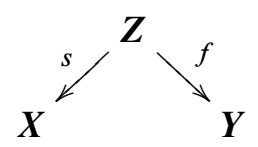


where $s \in \mathcal{~}$. Then $\alpha \circ \beta(\tilde{f})$ is given by the roof

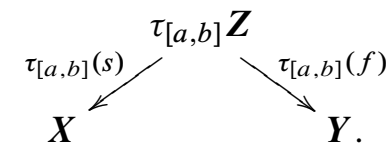

To show that this is equal to $\tilde{f}$ we construct a commutative diagram

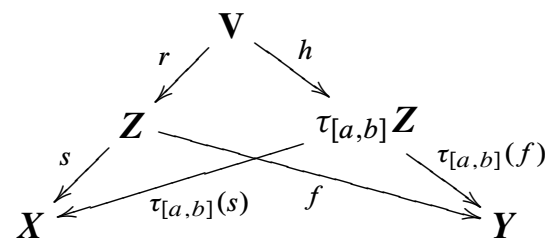

in which $s \circ r \in \mathcal{S}$. This diagram is easy to construct. Simply take $\boldsymbol{V}=\tau_{\leq b} \boldsymbol{Z}$ and let $r$ and $h$ be the unit and the counit of the adjunction for the functors $\tau_{\leq b}$ and $\tau_{\geq a}$, respectively.

The proof in the case of a torsion pair is exactly the same, once we take $\tau_{\geq a}, \tau_{\leq b}$ to be the truncation functors of the $t$-structure corresponding to the torsion pair.

\section{Decorated complexes in $A$}

Let $A$ be an abelian category. We will not fix a torsion pair on $A$ yet.

A decorated complex in A consists of the following data:

- a chain complex in $\mathrm{A}$

$$
E^{\bullet}: \cdots \stackrel{\delta}{\rightarrow} E^{n-1} \stackrel{\delta}{\rightarrow} E^{n} \stackrel{\delta}{\rightarrow} E^{n+1} \stackrel{\delta}{\rightarrow} \cdots
$$

- a graded subobject $M^{\bullet}$ of the underlying graded object of $E^{\bullet}$. That is, a sequence $M^{n} \subseteq E^{n}, n \in \mathbb{Z}$, of subobjects, not necessarily respected by $\delta$.

By a morphism $\left(E^{\bullet}, M^{\bullet}\right) \rightarrow\left(F^{\bullet}, N^{\bullet}\right)$ of decorated complexes we mean a map $f: E^{\bullet} \rightarrow F^{\bullet}$ of complexes such that $f\left(M^{n}\right) \subseteq N^{n}$ for every $n$.

We denote the category of decorated complexes by $\operatorname{Dec}(A)$.

5.1. Zero and full decorations. There are two natural decorations on every complex $E^{\bullet}$ : the zero decoration, in which all $M^{n}$ are zero, and the full decoration, in which $M^{n}=E^{n}$, for all $n$. We have the corresponding fully faithful embeddings

$$
\begin{aligned}
& i: \operatorname{Ch}(\mathrm{A}) \hookrightarrow \operatorname{Dec}(\mathrm{A}), \text { zero decoration, } \\
& j: \operatorname{Ch}(\mathrm{A}) \hookrightarrow \operatorname{Dec}(\mathrm{A}), \text { full decoration. }
\end{aligned}
$$


Both of these functors have both left and right adjoints. The left adjoint to $j$ is the forgetful functor (forgetting the decoration), and the right adjoint is what is denoted by $\mathscr{H}^{-1}$ in Section 5.2. The left adjoint to $i$ is $\mathscr{H}^{0}[-1]$, and the right adjoint to $i$ is the forgetful functor.

5.2. Various cohomologies associated to decorated complexes. Other than the usual cohomology of the complex $E^{\bullet}$, to a decorated complex $\left(E^{\bullet}, M^{\bullet}\right)$ we can associate the following two families of cohomologies:

$$
\begin{aligned}
H^{-1, n}\left(E^{\bullet}, M^{\bullet}\right) & :=\operatorname{ker}\left(M^{n} \stackrel{\delta}{\rightarrow} E^{n+1} / M^{n+1}\right), \\
H^{0, n}\left(E^{\bullet}, M^{\bullet}\right) & :=\operatorname{coker}\left(M^{n} \stackrel{\delta}{\rightarrow} E^{n+1} / M^{n+1}\right) .
\end{aligned}
$$

These cohomologies themselves fit into two complexes:

$$
\begin{aligned}
& \mathscr{H}^{-1}\left(E^{\bullet}, M^{\bullet}\right): \\
& \quad \cdots \rightarrow H^{-1, n-1}\left(E^{\bullet}, M^{\bullet}\right) \rightarrow H^{-1, n}\left(E^{\bullet}, M^{\bullet}\right) \rightarrow H^{-1, n+1}\left(E^{\bullet}, M^{\bullet}\right) \rightarrow \cdots, \\
& \mathcal{H}^{0}\left(E^{\bullet}, M^{\bullet}\right): \\
& \quad \cdots \rightarrow H^{0, n-1}\left(E^{\bullet}, M^{\bullet}\right) \rightarrow H^{0, n}\left(E^{\bullet}, M^{\bullet}\right) \rightarrow H^{0, n+1}\left(E^{\bullet}, M^{\bullet}\right) \rightarrow \cdots .
\end{aligned}
$$

Let us denote the cohomologies of these complexes by $H^{n}\left(\mathcal{H}^{0}\left(E^{\bullet}, M^{\bullet}\right)\right)$ and $H^{n}\left(\mathscr{H}^{-1}\left(E^{\bullet}, M^{\bullet}\right)\right)$, or by $H^{n}\left(\mathscr{H}^{-1}\right)$ and $H^{n}\left(\mathscr{H}^{0}\right)$ if $\left(E^{\bullet}, M^{\bullet}\right)$ is understood from the context.

Proposition 5.1. There are natural morphisms $H^{n-1}\left(\mathscr{H}^{0}\right) \stackrel{\partial}{\rightarrow} H^{n+1}\left(\mathscr{H}^{-1}\right)$ fitting in a long exact sequence

$$
\begin{aligned}
\cdots \rightarrow H^{n-2}\left(\mathscr{H}^{0}\right) \stackrel{\partial}{\rightarrow} H^{n}\left(\mathscr{H}^{-1}\right) \rightarrow H^{n}(E) \rightarrow H^{n-1}\left(\mathscr{H}^{0}\right) \stackrel{\partial}{\rightarrow} \\
\quad H^{n+1}\left(\mathscr{H}^{-1}\right) \rightarrow H^{n+1}(E) \rightarrow H^{n}\left(\mathscr{H}^{0}\right) \stackrel{\partial}{\rightarrow} H^{n+2}\left(\mathscr{H}^{-1}\right) \rightarrow \cdots .
\end{aligned}
$$

Proof. For simplicity, we denote $E^{\bullet}$ by $E$. We put a filtration on the complex $E$ by setting

$$
F^{n} E=M^{n} \oplus \bigoplus_{n<i} E^{i}
$$

This gives rise to a spectral sequence whose second page is exactly the union of the complexes $\mathscr{H}^{-1}$ and $\mathscr{H}^{0}$. The differentials of the third page are the morphisms $H^{n-1}\left(\mathscr{H}^{0}\right) \stackrel{\partial}{\rightarrow} H^{n+1}\left(\mathscr{H}^{-1}\right)$, and after the third page all the differential become zero for degree reasons. The fact that this spectral sequence converges to $H^{n}(E)$ is equivalent to the existence of the above long exact sequence.

An alternative proof can be obtained by showing that the natural map of complexes $E / \mathscr{H}^{-1} \rightarrow \mathscr{H}^{0}[-1]$ is a quasi-isomorphism. 
We define two classes of morphisms $\delta_{\text {sis }}$ and $\delta_{\text {qis }}$ in $\operatorname{Dec}(\mathrm{A})$. The former is the class of all morphisms in $\operatorname{Dec}(\mathrm{A})$ which induce isomorphisms on all $H^{i, n}, i=-1,0$, $n \in \mathbb{Z}$. The latter is the class of all quasi-isomorphisms, that is, all morphisms in $\operatorname{Dec}(\mathrm{A})$ which induce isomorphisms on the usual cohomologies $H^{n}$.

Proposition 5.2. We have $\oiint_{\text {sis }} \subset \wp_{\text {qis. }}$.

Proof. This follows from Proposition 5.1.

In Section 6 we will see the relation between the classes $\delta_{\text {sis }}$ and $\delta_{\text {qis }}$ in $\mathrm{Ch}^{\text {st }}(\mathrm{B})$, as defined in Section 4 , and the classes $\delta_{\text {sis }}$ and $\delta_{\text {qis }}$ defined above. Presumably $\boldsymbol{\delta}_{\text {sis }}$ is not a localizing class in $\operatorname{Dec}(\mathrm{A})$. However, its restriction to the full subcategory $\mathrm{Ch}(\mathrm{A}, \mathcal{T}, \mathcal{F})$ defined in Section 5.4 is a localizing class.

5.3. The cohomological functor $\mathbb{H}: \mathrm{Ch}(\mathrm{A}) \rightarrow \mathrm{B}$ associated to a torsion pair. Assume now that $A$ is equipped with a torsion pair $(\mathcal{T}, \mathcal{F})$. There is a cohomological functor $\mathbb{E}: \mathrm{Ch}(\mathrm{A}) \rightarrow \mathrm{B}$ obtained from the $t$-structure associated to the torsion pair $(\mathcal{T}, \mathcal{F})$. We give an explicit description of this cohomological functor.

Let $E^{\bullet}$ be a complex in $\mathrm{Ch}(\mathrm{A})$. We define $A^{n}, n \in \mathbb{Z}$, to be the subobject of $E^{n}$ such that

$$
\operatorname{im}\left(E^{n-1} \stackrel{\delta}{\rightarrow} E^{n}\right) \subseteq A^{n} \subseteq \operatorname{ker}\left(E^{n} \stackrel{\delta}{\rightarrow} E^{n+1}\right),
$$

and

$$
A^{n} / \operatorname{im}\left(E^{n-1} \stackrel{\delta}{\rightarrow} E^{n}\right) \in \mathcal{T} \quad \text { and } \quad \operatorname{ker}\left(E^{n} \stackrel{\delta}{\rightarrow} E^{n+1}\right) / A^{n} \in \mathcal{F} .
$$

These properties uniquely determine $A^{n}$.

For a chain complex $E^{\bullet}$ in $\mathrm{Ch}(\mathrm{A})$ we define

$$
\mathbb{Q}^{n}\left(E^{\bullet}\right):=\left[E^{n} / A^{n} \stackrel{\delta}{\rightarrow} A^{n+1}\right] \in \mathrm{B} .
$$

Proposition 5.3. Let $E^{\bullet}$ be a chain complex in $\mathrm{A}$. Then, for every $n$, there is a short exact sequence

$$
0 \rightarrow H^{0}\left(\mathbb{Q}^{n-1}\left(E^{\bullet}\right)\right) \rightarrow H^{n}\left(E^{\bullet}\right) \rightarrow H^{-1}\left(\mathbb{\mathbb { T } ^ { n }}\left(E^{\bullet}\right)\right) \rightarrow 0 .
$$

Note that $H^{0}\left(\mathbb{\square}^{n-1}\left(E^{\bullet}\right)\right) \in \mathcal{T}$ and $H^{-1}\left(\mathbb{M}^{n}\left(E^{\bullet}\right)\right) \in \mathcal{F}$.

Proof. This follows immediately from the definition of $\mathbb{\sharp}^{n}\left(E^{\bullet}\right)$.

To have a better grasp of the cohomological functor $\mathbb{t}$, it is perhaps useful to consider the two extreme cases in which $\mathcal{T}=\{0\}$ or $\mathcal{F}=\{0\}$. In both cases, we have a natural identification $\mathrm{B}=\mathrm{A}$. In the first case, $\mathbb{U}^{n}=H^{n}$, and in the second case $\mathbb{Q}^{n}=H^{n+1}$. For a general torsion pair, $\mathbb{t}^{n}$ has a little bit of $H^{n}$ and a little bit of $H^{n+1}$, as we saw in Proposition 5.3.

The next corollary follows immediately from Proposition 5.3. 
Corollary 5.4. A morphism $E^{\bullet} \rightarrow F^{\bullet}$ in $\mathrm{Ch}(\mathrm{A})$ is a quasi-isomorphism if and only if it induces isomorphisms on all $\mathbb{\boxplus}^{n}$.

5.4. Decorations in the presence of a torsion pair. Let $\left(E^{\bullet}, M^{\bullet}\right)$ be a decorated complex. We say that the decoration is compatible with the torsion pair $(\mathcal{T}, \mathcal{F})$ if the following condition is satisfied:

- For every $n, H^{-1, n}\left(E^{\bullet}, M^{\bullet}\right) \in \mathcal{F}$ and $H^{0, n}\left(E^{\bullet}, M^{\bullet}\right) \in \mathcal{T}$.

The decorated complexes whose decoration is compatible with the torsion pair form a full subcategory of $\operatorname{Dec}(\mathrm{A})$ which we denote by $\operatorname{Ch}(\mathrm{A}, \mathcal{T}, \mathcal{F})$. For an object $\left(E^{\bullet}, M^{\bullet}\right)$ in $\operatorname{Ch}(\mathrm{A}, \mathcal{T}, \mathcal{F})$ we define $\mathbb{H}^{n}\left(E^{\bullet}, M^{\bullet}\right)=\mathbb{H}^{n}\left(E^{\bullet}\right)$.

If we intersect the classes $\wp_{\text {sis }}, \oiint_{\text {qis }} \subset \operatorname{Dec}(\mathrm{A})$ defined in Section 5.2 with the subcategory $\operatorname{Ch}(A, \mathcal{T}, \mathcal{F})$, we obtain two classes of morphisms in $\operatorname{Ch}(A, \mathcal{T}, \mathcal{F})$, for which we use the same notation. The class $\delta_{\text {sis }}$ is a localizing class. This follows, for example, from Theorem 6.1 below.

We will see in Section 6 that there exists a natural equivalence of categories $\mathrm{Ch}^{\text {st }}(\mathrm{B}) \stackrel{\sim}{\longrightarrow} \mathrm{Ch}(\mathrm{A}, \mathcal{T}, \mathcal{F})$ under which the classes $\wp_{\text {sis }}, \wp_{\text {qis }} \subset \mathrm{Ch}^{\text {st }}(\mathrm{B})$ defined in Section 4 exactly correspond to the classes $\delta_{\text {sis }}, \boldsymbol{\vartheta}_{\text {qis }} \subset \mathrm{Ch}(\mathrm{A}, \mathcal{T}, \mathcal{F})$ defined above.

5.5. Homological algebra in $\operatorname{Dec}(\mathbf{A})$. The category $\operatorname{Dec}(A)$ should be thought of as a generalization of the category $\mathrm{Ch}(\mathrm{A})$ in the sense that we can do homological algebra in $\operatorname{Dec}(\mathrm{A})$. This means, the usual notions of homological algebra (such as, mapping cylinder of a morphism, mapping cone of a morphism, chain homotopy between morphisms, and so on) can be defined in $\operatorname{Dec}(A)$.

Let us show how mapping cylinders are defined in $\operatorname{Dec}(A)$. (Essentially, all other definitions can be formally reduced to this one.) Let $f:\left(E^{\bullet}, M^{\bullet}\right) \rightarrow\left(F^{\bullet}, N^{\bullet}\right)$ be a morphism in $\operatorname{Dec}(\mathrm{A})$ and denote $E^{\bullet} \rightarrow F^{\bullet}$ by $\bar{f}$. We define $\operatorname{Cyl}(f)$ to be the usual mapping cylinder $\operatorname{Cyl}(\bar{f})=E^{\bullet} \oplus E^{\bullet+1} \oplus F^{\bullet}$, endowed with the direct sum of the decorations of its components. We have natural morphisms $\left(E^{\bullet}, M^{\bullet}\right) \hookrightarrow \operatorname{Cyl}(f)$ and $\left(F^{\bullet}, N^{\bullet}\right) \hookrightarrow \operatorname{Cyl}(f)$ in $\operatorname{Dec}(\mathrm{A})$. In the case where $f$ is the identity, $\operatorname{Cyl}(f)$ is the cylinder of $\left(E^{\bullet}, M^{\bullet}\right)$, which can be used to define decorated chain homotopies. The quotient $\operatorname{Cone}(f):=\operatorname{Cyl}(f) /\left(E^{\bullet}, M^{\bullet}\right) \in \operatorname{Dec}(\mathrm{A})$ is the mapping cone of $f$, and so on.

Lemma 5.5. Let $f:\left(E^{\bullet}, M^{\bullet}\right) \rightarrow\left(F^{\bullet}, N^{\bullet}\right)$ be a morphism in $\operatorname{Dec}(\mathrm{A})$, and let Cone $(f)$ be its decorated cone as defined above. Then we have the isomorphisms

$$
H^{i, n}(\operatorname{Cone}(f)) \cong H^{i, n+1}\left(E^{\bullet}, M^{\bullet}\right) \oplus H^{i, n}\left(F^{\bullet}, N^{\bullet}\right), \quad i=-1,0 .
$$

Proof. Straightforward.

Remark 5.6. Decorated homotopies do not necessarily induce isomorphisms on $H^{i, n}$, but they induce chain homotopies on the level of complexes $\mathscr{H}^{-1}$ and $\mathscr{H}^{0}$. 
Passing to decorated chain homotopy classes of morphisms in $\operatorname{Dec}(A)$ we obtain the homotopy category $\mathrm{KDec}(\mathrm{A})$ of decorated chain complexes. This is a triangulated category with the usual shift functor. Doing the same with $\operatorname{Ch}(A, \mathcal{T}, \mathcal{F})$ we obtain a full triangulated subcategory of $\operatorname{KDec}(A)$ which we denote by $K(A, \mathcal{T}, \mathcal{F})$. (Here we have used the fact that the cone of a morphism in $\operatorname{Ch}(A, \mathcal{T}, \mathcal{F})$ is again in $\operatorname{Ch}(A, \mathcal{T}, \mathcal{F})$; see Lemma 5.5 above.)

The class $\delta_{\text {qis }} \subseteq \mathrm{KDec}(\mathrm{A})$ is a localizing class, and so is its intersection with $\mathrm{K}(\mathrm{A}, \mathcal{T}, \mathcal{F})$, for which we use the same notation $\boldsymbol{\delta}_{\text {qis }}$. This is because both are defined by a cohomological functor. More precisely, we are using the following.

Lemma 5.7 ([We], Proposition 10.4.1). Let $\mathrm{K}$ be a triangulated category, and let 8 be the class of quasi-isomorphisms with respect to a certain cohomological functor. Then 8 is a localizing class.

As in classical homological algebra, the fact that $\delta_{\text {qis }}$ becomes a localizing class is very useful. Note that

$$
\delta_{\mathrm{qis}}^{-1} \mathrm{KDec}(\mathrm{A}) \cong \oint_{\mathrm{qis}}^{-1} \operatorname{Dec}(\mathrm{A}) \text { and } \quad \delta_{\mathrm{qis}}^{-1} \mathrm{~K}(\mathrm{~A}, \mathcal{T}, \mathcal{F}) \cong \oint_{\mathrm{qis}}^{-1} \operatorname{Ch}(\mathrm{A}, \mathcal{T}, \mathcal{F}) .
$$

There are two other triangulated subcategories of $K(A, \mathcal{T}, \mathcal{F})$ that are less important for us (they will only be used in the proof of Theorem 7.6). We will end this section by giving their definitions. The first category, denoted $F(A, \mathcal{T}, \mathcal{F})$, is the full subcategory of $\mathrm{K}(\mathrm{A}, \mathcal{T}, \mathcal{F})$ consisting of decorated complexes $\left(E^{\bullet}, M^{\bullet}\right)$ such that $E^{\bullet}$ is a complex of free objects, i.e., $E^{n} \in \mathcal{F}$ for all $n$. This is a triangulated category because the cone of morphism between two complexes of free objects is again a complex of free objects.

The category $\operatorname{F}(A, \mathcal{T}, \mathcal{F})$ contains a subcategory $\operatorname{FF}(A, \mathcal{T}, \mathcal{F})$ consisting of the complexes with the full decoration. We will need the following lemmas for the proof of Theorem 7.6.

Lemma 5.8. Let $\mathrm{K}$ be a triangulated category, and let 8 a localizing class in $\mathrm{K}$ that is defined by a cohomological functor. Let $\mathrm{F}$ be a full triangulated subcategory of $\mathrm{K}$, and set $\delta_{\mathrm{F}}=\delta \cap \mathrm{F}$. Assume either of the following holds:

- for every $X \in \mathrm{K}$, there exists a quasi-isomorphism $F \rightarrow X$ with $F \in \mathrm{F}$;

- for every $X \in \mathrm{K}$, there exists a quasi-isomorphism $X \rightarrow F$ with $F \in \mathrm{F}$.

Then the functor $\delta_{\mathrm{F}}^{-1} \mathrm{~F} \rightarrow \delta^{-1} \mathrm{~K}$ is an equivalence of triangulated categories.

Proof. Essential surjectivity is obvious, so it is enough to show that $\mathrm{F}$ is a localizing subcategory of K. This follows from [GeMa], Proposition III.2.10 (p. 151). (Note that, by Lemma $5.7, \delta_{\mathrm{F}}$ is automatically a localizing class.) 
Lemma 5.9. The inclusion $\operatorname{FF}(A, \mathcal{T}, \mathcal{F}) \subset \mathrm{F}(\mathrm{A}, \mathcal{T}, \mathcal{F})$ induces an equivalence of triangulated categories

$$
\S_{\mathrm{qis}}^{-1} \mathrm{FF}(\mathrm{A}, \mathcal{T}, \mathcal{F}) \cong \oint_{\mathrm{qis}}^{-1} \mathrm{~F}(\mathrm{~A}, \mathcal{T}, \mathcal{F}) .
$$

Proof. For every $\left(E^{\bullet}, M^{\bullet}\right)$ in $\mathrm{F}(\mathrm{A}, \mathcal{T}, \mathcal{F})$ we have a quasi-isomorphism $\left(E^{\bullet}, M^{\bullet}\right) \rightarrow$ $\left(E^{\bullet}, E^{\bullet}\right)$, where $\left(E^{\bullet}, E^{\bullet}\right) \in \operatorname{FF}(\mathrm{A}, \mathcal{T}, \mathcal{F})$. The result follows from the second case of Lemma 5.8 .

Dually, there are triangulated subcategories

$$
\mathrm{ZT}(\mathrm{A}, \mathcal{T}, \mathcal{F}) \subset \mathrm{T}(\mathrm{A}, \mathcal{T}, \mathcal{F}) \subset \mathrm{K}(\mathrm{A}, \mathcal{T}, \mathcal{F}),
$$

where $T(A, \mathcal{T}, \mathcal{F})$ consists of decorated complexes of torsion objects and $Z T(A, \mathcal{T}, \mathcal{F})$ is its full subcategory consisting of complexes with zero decoration. The above discussion applies to these categories as well.

Remark 5.10. The discussion of this subsection applies to the case where the complexes are bounded (above, below, or both).

\section{Complexes in B and the derived category $\mathscr{D}(B)$}

In this section we give an alternative description of $\mathrm{Ch}^{\text {st }}(\mathrm{B})$; see Theorem 6.1. This, together with Proposition 4.3, enables us to give a simple description for the derived category $\mathscr{D}(\mathrm{B})$.

6.1. Description of $\mathrm{Ch}(\mathrm{B})$ via decorated complexes. The main result of this section is the following.

Theorem 6.1. There is an equivalence of categories

$$
\text { Tot: } \mathrm{Ch}^{\text {st }}(\mathrm{B}) \stackrel{\sim}{\longrightarrow} \mathrm{Ch}(\mathrm{A}, \mathcal{T}, \mathcal{F}) .
$$

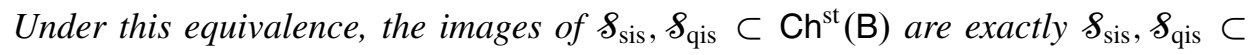
$\operatorname{Ch}(\mathrm{A}, \mathcal{T}, \mathcal{F})$.

Recall that $\oiint_{\text {qis }} \subset \mathrm{Ch}(\mathrm{A}, \mathcal{T}, \mathcal{F})$ is the class of all quasi-isomorphisms, that is, all morphisms $\left(E^{\bullet}, M^{\bullet}\right) \rightarrow\left(F^{\bullet}, N^{\bullet}\right)$ such that $E^{\bullet} \rightarrow F^{\bullet}$ is a quasi-isomorphism in $\mathrm{Ch}(\mathrm{A})$. The class $\delta_{\text {sis }} \subset \delta_{\text {qis }}$ consists of those morphisms $\left(E^{\bullet}, M^{\bullet}\right) \rightarrow\left(F^{\bullet}, N^{\bullet}\right)$ which induce isomorphisms on $\mathscr{H}^{-1}$ and $\mathscr{H}^{0}$; see Section 5.2.

The following corollary is immediate from Theorem 6.1. 
Corollary 6.2. The functor Tot induces an equivalence of categories:

$$
\begin{aligned}
& \mathrm{Ch}(\mathrm{B}) \stackrel{\sim}{\longrightarrow} \delta_{\text {sis }}^{-1} \mathrm{Ch}(\mathrm{A}, \mathcal{T}, \mathcal{F}), \\
& \mathcal{D}(\mathrm{B}) \stackrel{\sim}{\longrightarrow} \delta_{\text {qis }}^{-1} \mathrm{Ch}(\mathrm{A}, \mathcal{T}, \mathcal{F}) .
\end{aligned}
$$

Proof. Follows from Proposition 4.3.

We prove Theorem 6.1 by giving a step by step of simplification of what goes into the definition of a chain complex in B. We begin by complexes of length two.

6.2. Complexes of length 2 in $B$. Consider the morphisms $P: \mathbb{X} \rightarrow Y$ and $Q: Y \rightarrow$ $\mathbb{Z}$ as in the following diagram:

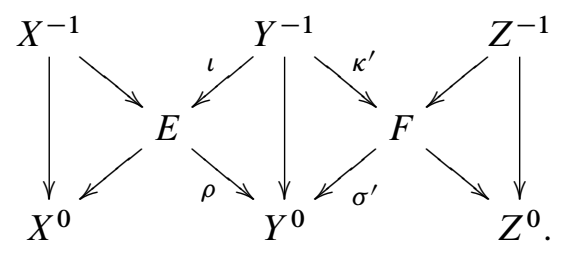

Let $A \subseteq E$ be defined as in Section 3.4, and let $B \subseteq F$ be the corresponding object for the morphism $Q$.

Lemma 6.3. The composition $Q \circ P$ is zero if and only if there is a morphism $f: E / A \rightarrow B$ in A making the following diagram commute:

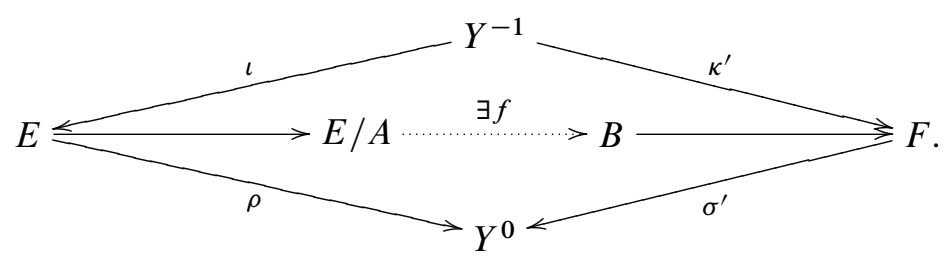

In this case, the monomorphism im $P \rightarrow \operatorname{ker} Q$ is realized by the strict morphism

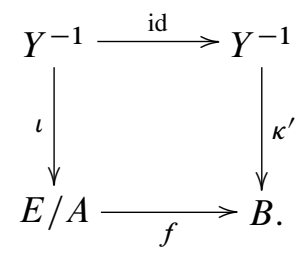

Proof. Recall from Section 3.4 and Section 3.5 that im $P=\left[Y^{-1} \rightarrow E / A\right]$ and $\operatorname{ker} Q=\left[Y^{-1} \rightarrow B\right]$. The composition $Q \circ P$ being zero is equivalent to the 
existence of a commutative triangle

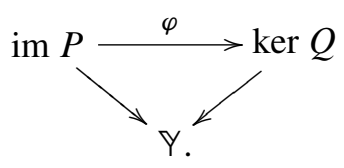

If we unravel this triangle, we see that it is equivalent to the diagram required in the lemma.

Corollary 6.4. In the sequence $\mathbb{X} \stackrel{P}{\rightarrow} Y \stackrel{Q}{\rightarrow} \mathbb{Z}$ above the cohomology at $Y$ is given by

$$
\mathbb{H}:=[E / A \stackrel{f}{\rightarrow} B] .
$$

Corollary 6.5. A sequence $\mathbb{X} \stackrel{P}{\rightarrow} Y \stackrel{Q}{\rightarrow} \mathbb{Z}$ as above is exact at $Y$ if and only if there is an isomorphism $E / A \stackrel{\sim}{\longrightarrow} B$ respecting the morphisms $\iota, \rho, \kappa^{\prime}$, and $\sigma^{\prime}$.

6.3. An alternative way of looking at complexes in B. Let $P$ and $Q$ be as in Section 6.2. By a link from $P$ to $Q$ we mean a morphism $\delta: E \rightarrow F$ such that:

(L) The following diagram commutes and the horizontal sequence is a complex:

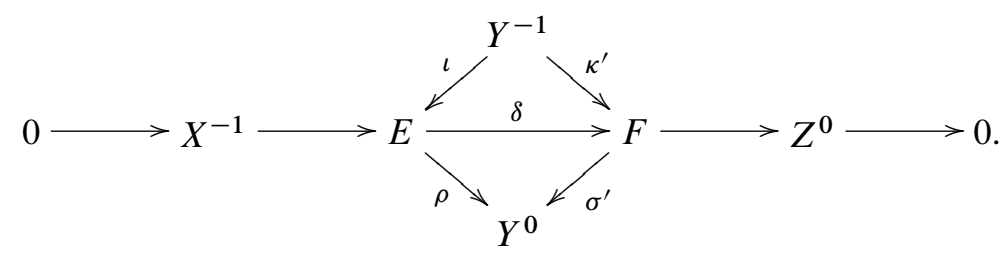

Proposition 6.6. Consider the sequence $\mathbb{X} \stackrel{P}{\rightarrow} Y \stackrel{Q}{\rightarrow} \mathbb{Z}$. Then $Q \circ P=0$ if and only if there exists a link from $P$ to $Q$. If such a link exists then it is unique.

Proof. One implication is trivial from Lemma 6.3. To prove the reverse implication, let $A \subseteq E$ and $B \subseteq F$ be as in Lemma 6.3. We need to show that every $\delta: E \rightarrow F$ as in (L) necessarily vanishes on $A$ and factors through $B$; the result will then follow from Lemma 6.3.

Let us prove that $\delta$ vanishes on $A$. Since $\delta\left(X^{-1}\right)=0$, we have an induced map $\bar{\delta}: H^{-1}(C(P)) \rightarrow F$. By definition, the image $T$ of $A$ in $H^{-1}(C(P))$ is the torsion part of $H^{-1}(C(P))$. Observe that $\bar{\delta}: H^{-1}(C(P)) \rightarrow F$ factors through the kernels of both $\sigma^{\prime}: F \rightarrow Y^{0}$ and $\rho^{\prime}: F \rightarrow Z^{0}$, and that $\operatorname{ker} \sigma^{\prime} \cap \operatorname{ker} \rho^{\prime}=\operatorname{ker}\left(d: Z^{-1} \rightarrow Z^{0}\right)$ belongs to $\mathcal{F}$. So $\bar{\delta}(T)=0$. That is $\delta(A)=0$.

The proof that $\delta$ factors through $B$ is similar. 
We now prove the uniqueness. Let $\delta$ and $\delta^{\prime}$ be two links, and set $\epsilon=\delta-\delta^{\prime}$. By the commutativity condition of (L), $\epsilon$ vanishes on $Y^{-1}$. Since the horizontal sequence is a complex, $\epsilon$ vanishes on $X^{-1}$ as well. Hence, $\epsilon$ factors through $E /\left(\kappa\left(X^{-1}\right)+\right.$ $\iota\left(Y^{-1}\right) \cong H^{0}(\mathbb{X})$.

Similarly, by the commutativity condition of $(\mathrm{L}), \sigma^{\prime} \circ \epsilon=0$, and since the horizontal sequence is a complex, $\rho^{\prime} \circ \epsilon=0$, where $\rho^{\prime}$ is the morphism $\rho^{\prime}: F \rightarrow Z^{0}$. This implies that $\epsilon$ factors through $\operatorname{ker} \rho^{\prime} \cap \operatorname{ker} \sigma^{\prime} \cong H^{-1}(\mathbb{Z})$.

Putting these together, we see that $\epsilon$ factors through a map $H^{0}(\mathbb{X}) \rightarrow H^{-1}(\mathbb{Z})$. But such a map is necessarily zero because $H^{0}(\mathbb{X})$ is in $\mathcal{T}$ and $H^{-1}(\mathbb{Z})$ is in $\mathcal{F}$. Therefore, $\epsilon$ is the zero map. That is, $\delta=\delta^{\prime}$.

Proposition 6.7. The complex

$$
0 \rightarrow \mathbb{X} \stackrel{P}{\rightarrow} \mathrm{Y} \stackrel{Q}{\rightarrow} \mathbb{Z} \rightarrow 0
$$

is short exact if and only of the middle sequence in $(\mathrm{L})$ is exact, $H^{-1}(C(P)) \in \mathcal{F}$, and $H^{-1}(C(Q)) \in \mathcal{T}$.

Proof. Follows from Corollary 3.3 and Corollary 6.4.

Using the idea of link, we see that a chain complex

$$
\boldsymbol{X}=\cdots \rightarrow{ }^{n-2} \mathbb{X} \rightarrow{ }^{n-1} \mathbb{X} \rightarrow{ }^{n} \mathbb{X} \rightarrow{ }^{n+1} \mathbb{X} \rightarrow{ }^{n+2} \mathbb{X} \rightarrow \cdots,
$$

with ${ }^{n} \mathbb{X}=\left[d:{ }^{n} X^{-1} \rightarrow{ }^{n} X^{0}\right]$, is equivalently described by the diagram

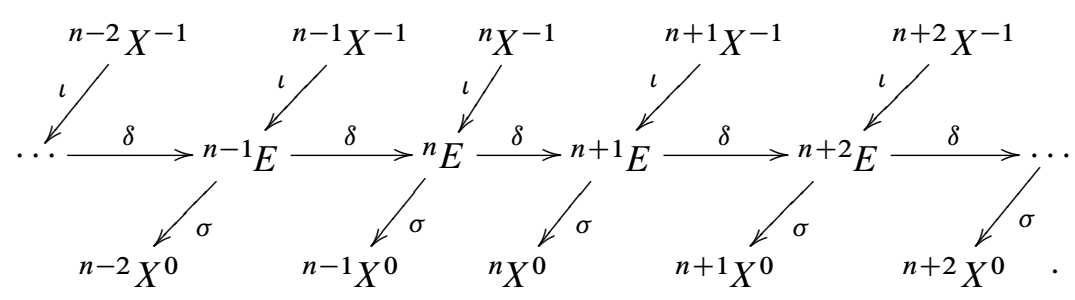

Literally translating all the commutativity and exactness conditions that are needed to be satisfied, we arrive at the following list of requirements:

(1) every NW-SE sequence is short exact;

(2) $\sigma \circ \delta \circ \iota=d$;

(3) $\delta^{2} \circ \iota=0$ and $\sigma \circ \delta^{2}=0$.

The third axiom can be improved though.

Lemma 6.8. We have $\delta^{2}=0$. 
Proof. Let ${ }^{n} C:=\operatorname{ker}(\delta \circ \iota) / \operatorname{im}(\sigma \circ \delta)$, i.e., the middle cohomology of the sequence

$$
{ }^{n-1} X^{-1} \stackrel{\delta \circ \iota}{\longrightarrow}{ }^{n} E \stackrel{\sigma \circ \delta}{\longrightarrow}{ }^{n} X^{0},
$$

that is a complex by (3). Let ${ }^{n} A:=q^{-1}(T) \subseteq{ }^{n} E$, where $T \subseteq{ }^{n} C$ is the torsion part of ${ }^{n} C$. As we saw in the proof of Proposition 6.6, $\delta:{ }^{n-1} E \rightarrow{ }^{n} E$ factors through ${ }^{n} A$, and $\delta:{ }^{n} E \rightarrow{ }^{n+1} E$ vanishes on ${ }^{n} A$. This means that

$$
\delta\left({ }^{n-1} E\right) \subseteq{ }^{n} A \subseteq \operatorname{ker}\left({ }^{n} E \stackrel{\delta}{\rightarrow}{ }^{n+1} E\right) .
$$

Therefore, $\delta^{2}=0$.

Summarizing the above discussion, to give a complex $\boldsymbol{X}$ in B is equivalent to giving a diagram as above such that (1) and (2) are satisfied. Conditions (1) and (2) are saying that the objects ${ }^{n} X^{0}$ are redundant and can be deduced from the rest of the data. So we can scrape off all the ${ }^{n} X^{0}$, hence also the axioms (1) and (2), without losing any information about $\boldsymbol{X}$. This leads to the definition of the functor Tot: $\mathrm{Ch}^{\text {st }}(\mathrm{B}) \rightarrow \mathrm{Ch}(\mathrm{A}, \mathcal{T}, \mathcal{F})$ that is discussed in the nest subsection.

6.4. Definition of the functor Tot. We define the functor

$$
\text { Tot: } \mathrm{Ch}^{\mathrm{st}}(\mathrm{B}) \rightarrow \operatorname{Ch}(\mathrm{A}, \mathcal{T}, \mathcal{F})
$$

as follows. Let $\boldsymbol{X}$ be a complex in B as in Section 6.3. We set $\operatorname{Tot}(\boldsymbol{X})=\left(E^{\bullet}, M^{\bullet}\right)$, where $E^{n}:={ }^{n} E$ and $M^{n}:=\iota\left({ }^{n} X^{-1}\right)$.

To define the effect of Tot on morphisms, let $\boldsymbol{X}$ and $\boldsymbol{Y}$ be complexes in B, and let $\operatorname{Tot}(\boldsymbol{X})=\left(E^{\bullet}, M^{\bullet}\right)$ and $\operatorname{Tot}(\boldsymbol{Y})=\left(F^{\bullet}, N^{\bullet}\right)$. If we use Tot and literally translate what goes into the definition of a morphism of complexes $\boldsymbol{X} \rightarrow \boldsymbol{Y}$ in $\mathrm{Ch}^{\text {st }}$ (B), we see that such a morphism is given by a collection of morphisms $f_{n}: E^{n} \rightarrow F^{n}$ in $\mathrm{A}$ satisfying the following conditions:

(i) for every $n, f_{n}\left(M^{n}\right) \subseteq N^{n}$;

(ii) for every $n$, the "commutator"

$$
\epsilon_{n}:=f_{n+1} \circ \delta-\delta \circ f_{n}: E^{n} \rightarrow F^{n+1}
$$

vanishes on $M^{n}$ and factors through $N^{n+1}$.

There are two problems here, however. The first problem is that, a priori, this is not quite the same thing as a morphism in $\operatorname{Ch}(A, \mathcal{T}, \mathcal{F})$; we need that $\epsilon_{n}$ be actually equal to zero. This is shown to be the case in Lemma 6.9 below. The second problem is that a morphism $\boldsymbol{X} \rightarrow \boldsymbol{Y}$ does not, a priori, uniquely determine the collection $\left\{f_{n}\right\}$. It only uniquely determines the effect of $f_{n}$ on $M^{n}$ and $E^{n} / M^{n}$, but not on $E^{n}$. This is taken care of in Lemma 6.10 below.

The idea of the proof of both lemmas is very similar to the proof of Proposition 6.6. 
Lemma 6.9. For every $n$, we have $\epsilon_{n}=0$. That is, the diagrams

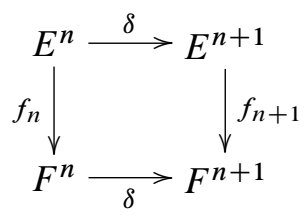

commute.

Proof. Note that, by definition, $E^{n}:={ }^{n} E$ and $M^{n}:=\iota\left({ }^{n} X^{-1}\right), F^{n}:={ }^{n} F$ and $N^{n}:=\iota\left({ }^{n} X^{-1}\right)$.

Condition (ii) implies that the diagram commutes when restricted to $M^{n} \subseteq E^{n}$. It also implies that the diagram commutes when composed with the quotient map $q_{n+1}: F^{n+1} \rightarrow F^{n+1} / N^{n+1}$.

If we use (i) and (ii) with $n-1$, it follows that the diagram commutes when restricted to $\delta\left(M^{n-1}\right)$ as well. That is, $\epsilon_{n}$ vanishes on $\delta\left(M^{n-1}\right)$. Therefore, $\epsilon_{n}$ induces a morphism

$$
\varphi: \operatorname{coker}\left(M^{n-1} \stackrel{p_{n} \circ \delta}{\longrightarrow} E^{n} / M^{n}\right) \rightarrow N^{n+1},
$$

where $p_{n}: E^{n} \rightarrow E^{n} / M^{n}$ is the quotient map.

Similarly, if we use (i) and (ii) with $n+1$, it follows that the diagram commutes when composed with $q_{n+2} \circ \delta: F^{n+1} \rightarrow F^{n+2} / N^{n+2}$. This implies that $\epsilon_{n}$, hence also $\varphi$, factors through the kernel of $N^{n+1} \rightarrow F^{n+2} / N^{n+2}$. In other words, we obtain a morphism

$$
\varphi: \operatorname{coker}\left(M^{n-1} \stackrel{p_{n} \circ \delta}{\longrightarrow} E^{n} / M^{n}\right) \rightarrow \operatorname{ker}\left(N^{n+1} \stackrel{q_{n+2} \circ \delta}{\longrightarrow} F^{n+2} / N^{n+2}\right) .
$$

Now observe that the left-hand side is equal to $H^{0}\left({ }^{n-1} \boldsymbol{X}\right) \in \mathcal{T}$, and the right-hand side is equal to $H^{-1}\left({ }^{n+1} \boldsymbol{X}\right) \in \mathcal{F}$. By the definition of a torsion pair, $\varphi$ has to be the zero map. This implies that $\epsilon_{n}=0$.

Lemma 6.10. Let $\left\{f_{n}\right\}$ and $\left\{f_{n}^{\prime}\right\}$ be two families of morphisms as above such that:

- for every $n, f_{n}=f_{n}^{\prime}: M^{n} \rightarrow N^{n}$

- for every $n, f_{n}=f_{n}^{\prime}: E^{n} / M^{n} \rightarrow F^{n} / N^{n}$.

Then $f_{n}=f_{n}^{\prime}$ for all $n$.

Proof. The proof is similar to the proof of the previous lemma. We set $h_{n}:=f_{n}-f_{n}^{\prime}$. By the first condition, $f_{n}$ and $f_{n}^{\prime}$ coincide on $M^{n}$. They also coincide on $d\left(M^{n-1}\right)$ by the previous lemma. So $h_{n}$ factors through $E^{n} /\left(M^{n}+d M^{n-1}\right)=H^{0}\left({ }^{n-1} \mathbb{X}\right)$. 
By the second condition, $h_{n}$ factors through $N^{n}$. It follows from the previous lemma that $d \circ h_{n}$ factors through $N^{n+1}$. That is, $h^{n}$ factors through $N^{n} \cap d^{-1}\left(N^{n+1}\right)=H^{-1}\left({ }^{n} \mathbb{X}\right)$.

Putting the above information together, we see that $h_{n}$ factors through a map $H^{0}\left({ }^{n-1} \mathbb{X}\right) \rightarrow H^{-1}\left({ }^{n} \mathbb{X}\right)$. By the properties of a torsion pair, $h_{n}$ is necessarily the zero map.

We finally come to the proof of Theorem 6.1.

Proof of Theorem 6.1. We define the inverse $G: \operatorname{Ch}(\mathrm{A}, \mathcal{T}, \mathcal{F}) \rightarrow \mathrm{Ch}^{\text {st }}(\mathrm{B})$ of Tot as follows. Given $\left(E^{\bullet}, M^{\bullet}\right) \in \operatorname{Ch}(\mathrm{A}, \mathcal{T}, \mathcal{F})$, we define $G\left(E^{\bullet}, M^{\bullet}\right) \in \mathrm{Ch}^{\text {st }}(\mathrm{B})$ to be the complex whose $n^{\text {th }}$ term is

$$
{ }^{n} \mathbb{X}:=\left[M^{n} \stackrel{p_{n+1} \circ \delta}{\longrightarrow} E^{n+1} / M^{n+1}\right] .
$$

Here $p_{n+1}: E^{n+1} \rightarrow E^{n+1} / M^{n+1}$ stands for the projection map. The differential $d:{ }^{n-1} \mathbb{X} \rightarrow{ }^{n} \mathbb{X}$ is the morphism defined by $E^{n}$. More precisely, it is given by the diagram

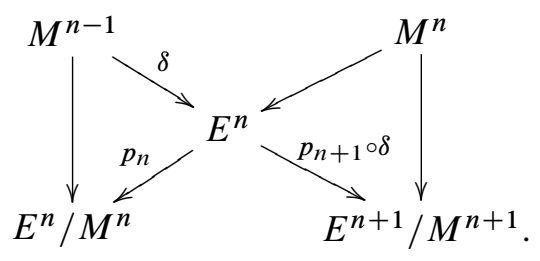

The arguments of this and the previous subsection can be reversed, in a trivial manner, to show that $G$ is an inverse to Tot.

We are not quite done yet with the proof of Theorem 6.1, because we have to show that the functors Tot and $G$ respect $\delta_{\text {sis }}$ and $\delta_{\text {qis }}$. We do this in the next section.

\section{Effect of Tot on derived categories}

In this section we study the effect of Tot on various cohomology groups and complete the proof of Theorem 6.1 by showing that Tot respects $\delta_{\text {sis }}$ and $\delta_{\text {qis }}$. As a corollary of this, we reprove a theorem of Happel-Reiten-Smalø asserting that in the case where the torsion pair is tilting or cotilting there is an equivalence of derived categories $\mathscr{D}(\mathrm{B}) \stackrel{\longrightarrow}{\longrightarrow} \mathscr{D}(\mathrm{A})$; see Theorem 7.6. 
7.1. Effect of Tot on cohomologies. Let $X$ be a complex in B. We denote the $n^{\text {th }}$ cohomology of $\boldsymbol{X}$ by

$$
\mathbb{Q}^{n}(\boldsymbol{X}):=\operatorname{im}^{n-1} d / \operatorname{ker}^{n} d .
$$

(Not to be confused with hypercohomology.) The rest of the notation appearing in the next proposition have been introduced in Section 5.2.

Proposition 7.1. For every $\boldsymbol{X}$ in $\mathrm{Ch}(\mathrm{B})$, we have natural isomorphisms

$$
\begin{aligned}
H^{-1, n}(\operatorname{Tot}(\boldsymbol{X})) & \cong H^{-1}\left({ }^{n} \mathbb{X}\right), \\
H^{0, n}(\operatorname{Tot}(\boldsymbol{X})) & \cong H^{0}\left({ }^{n} \mathbb{X}\right), \\
\mathbb{\square} \mathbb{D}^{n}(\operatorname{Tot}(\boldsymbol{X})) & \cong \mathbb{U}^{n}(\boldsymbol{X}) .
\end{aligned}
$$

Proof. The first two isomorphisms follow from the definition of Tot. The last one is simply a rephrasing of Corollary 6.4 .

Corollary 7.2. The functor $\mathrm{Tot}: \mathrm{Ch}^{\mathrm{st}}(\mathrm{B}) \rightarrow \mathrm{Ch}(\mathrm{A}, \mathcal{T}, \mathcal{F})$ maps $\boldsymbol{\delta}_{\mathrm{sis}}, \boldsymbol{8}_{\mathrm{qis}} \subset \mathrm{Ch}^{\mathrm{st}}(\mathrm{B})$ isomorphically to $\boldsymbol{\delta}_{\mathrm{sis}}, \boldsymbol{S}_{\mathrm{qis}} \subset \mathrm{Ch}(\mathrm{A}, \mathcal{T}, \mathcal{F})$. In particular, the functor Tot preserves, and reflects, quasi-isomorphisms.

Proof. Immediate.

7.2. Effect of Tot on derived categories. On $\operatorname{Ch}(A, \mathcal{T}, \mathcal{F})$ the shift functor $\left(E^{\bullet}, M^{\bullet}\right) \mapsto\left(E^{\bullet}[1], M^{\bullet}[1]\right)$ makes the localized category $\mathcal{\gamma}_{\text {qis }}^{-1} \operatorname{Ch}(\mathrm{A}, \mathcal{T}, \mathcal{F})$ into a triangulated category. We have the following.

Theorem 7.3. The functor $\mathrm{Tot}: \mathrm{Ch}^{\mathrm{st}}(\mathrm{B}) \rightarrow \mathrm{Ch}(\mathrm{A}, \mathcal{T}, \mathcal{F})$ induces a triangle equivalence

$$
\text { Tot: } \mathscr{D}(\mathrm{B}) \stackrel{\sim}{\rightarrow} \mathcal{8}_{\mathrm{qis}}^{-1} \mathrm{Ch}(\mathrm{A}, \mathcal{T}, \mathcal{F}) .
$$

In particular, we have equivalences of bounded derived categories

$$
\text { Tot: } \mathscr{D}^{*}(\mathrm{~B}) \stackrel{\sim}{\longrightarrow} \mathcal{S}_{\mathrm{qis}}^{-1} \mathrm{Ch}^{*}(\mathrm{~A}, \mathcal{T}, \mathcal{F}),
$$

where $*=-,+, b$.

Proof. This follows from Proposition 4.3 and Corollary 7.2. Note that Tot respects any kind of boundedness.

7.3. The forgetful functor $\Phi: \mathscr{D}(B) \rightarrow \mathscr{D}(\mathbf{A})$. We have a forgetful triangle functor

$$
\Phi: \delta_{\text {qis }}^{-1} \operatorname{Ch}(\mathrm{A}, \mathcal{T}, \mathcal{F}) \rightarrow \mathscr{D}(\mathrm{A}), \quad\left(E^{\bullet}, M^{\bullet}\right) \mapsto E^{\bullet} .
$$


By Theorem 7.3, this induces a triangle functor

$$
\Phi \circ \text { Tot: } \mathscr{D}(\mathrm{B}) \rightarrow \mathscr{D}(\mathrm{A}) \text {. }
$$

Furthermore, by Proposition 7.1, the following diagram commutes:

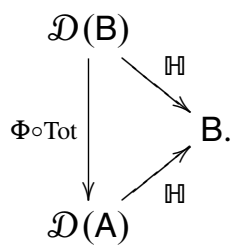

Here, the upper $\mathbb{H}$ stands for the usual cohomology of chain complexes, and the lower $\mathbb{H}$ is the cohomological functor defined in Section 5.3. The following is immediate.

Proposition 7.4. The functor $\Phi \circ$ Tot: $\mathscr{D}(\mathrm{B}) \rightarrow \mathscr{D}(\mathrm{A})$ reflects isomorphisms.

7.4. The equivalence $\mathscr{D}(\mathbf{B}) \stackrel{\sim}{\longrightarrow} \mathscr{D}(\mathbf{A})$. It is a theorem of Happel-Reiten-Smal $\varnothing$ that, in the case where $(\mathcal{T}, \mathcal{F})$ is either tilting or cotilting, there is an equivalence of bounded derived categories $\mathscr{D}^{b}(\mathrm{~B}) \rightarrow \mathscr{D}^{b}(\mathrm{~A}){ }^{1}$

We reprove this result using our approach. Recall that a torsion theory $(\mathcal{T}, \mathcal{F})$ is called cotilting if for every $A \in \mathrm{A}$ there exists an epimorphism $F \rightarrow A$ with $F \in \mathcal{F}$. The torsion theory is called tilting if for every $A \in \mathrm{A}$ there exists a monomorphism $A \rightarrow T$ with $T \in \mathcal{T}$.

Before proving the theorem, we show a lemma.

Lemma 7.5. Let $\left(E^{\bullet}, M^{\bullet}\right) \in \mathrm{Ch}(\mathrm{A}, \mathcal{T}, \mathcal{F})$, and let $f: F^{\bullet} \rightarrow E^{\bullet}$ be a morphism of complexes. Assume that for every $n, f^{n}: F^{n} \rightarrow E^{n}$ is an epimorphism with kernel in $\mathcal{F}$. Then the induced decoration $f^{-1}\left(M^{\bullet}\right)$ on $F^{\bullet}$ is compatible with $(\mathcal{T}, \mathcal{F})$.

Proof. Since $H^{-1, n}\left(F^{\bullet}, f^{-1}\left(M^{\bullet}\right)\right)$ is an extension of $H^{-1, n}\left(E^{\bullet}, M^{\bullet}\right)$ with $\operatorname{ker} f^{n}$, it belongs to $\mathcal{F}$ by Lemma 2.3. It is easy to see that $H^{0, n}\left(F^{\bullet}, f^{-1}\left(M^{\bullet}\right)\right) \cong$ $H^{0, n}\left(E^{\bullet}, M^{\bullet}\right)$, so it is in $\mathcal{T}$.

Theorem 7.6 (Happel-Reiten-Smalø). Assume $(\mathcal{T}, \mathcal{F})$ is cotilting (respectively, tilting). Then the functor

$$
\Phi \circ \text { Tot: } \mathscr{D}^{*}(\mathrm{~B}) \rightarrow \mathscr{D}^{*}(\mathrm{~A}), \quad *=-, b(\text { respectively, } *=+, b),
$$

defined in Section 7.3 is an equivalence of triangulated categories.

\footnotetext{
${ }^{1} \mathrm{In}[\mathrm{HaReSm}]$ they actually assume existence of enough injectives/projectives, but this is not necessary.
} 
Proof. We prove the claim in the cotilting case, so let $*=-$ or $*=b$. By Theorem 7.3, it is enough to show that the forgetful functor $\Phi: \delta_{\text {qis }}^{-1} \operatorname{Ch}(\mathrm{A}, \mathcal{T}, \mathcal{F}) \rightarrow \mathscr{D}(\mathrm{A})$ is an equivalence.

Let $\mathrm{FF}^{*}(A, \mathcal{T}, \mathcal{F}) \subset F^{*}(A, \mathcal{T}, \mathcal{F})$ be as in Section 5.5. Let $F^{*}(A)$ be the full subcategory of the homotopy category $\mathrm{K}^{*}(\mathrm{~A})$ of chain complexes in $\mathrm{A}$ consisting of complexes whose terms are in $\mathcal{F}$. Clearly $\Phi$ induces an equivalence $\operatorname{FF}^{*}(A, \mathcal{T}, \mathcal{F}) \stackrel{\sim}{\longrightarrow}$ $\mathrm{F}^{*}(\mathrm{~A})$ of triangulated categories, so, in particular, it induces an equivalence of the localized categories $\oint_{\text {qis }}^{-1} \mathrm{FF}^{*}(\mathrm{~A}, \mathcal{T}, \mathcal{F}) \stackrel{\sim}{\longrightarrow} \wp_{\text {qis }}^{-1} \mathrm{~F}^{*}(\mathrm{~A})$. To prove the result, we show that the functors

$$
\oint_{\mathrm{qis}}^{-1} \mathrm{FF}^{*}(\mathrm{~A}, \mathcal{T}, \mathcal{F}) \rightarrow \oint_{\mathrm{qis}}^{-1} \mathrm{Ch}^{*}(\mathrm{~A}, \mathcal{T}, \mathcal{F}) \quad \text { and } \quad \delta_{\mathrm{qis}}^{-1} \mathrm{~F}^{*}(\mathrm{~A}) \rightarrow \mathscr{D}^{*}(\mathrm{~A}),
$$

induced by the corresponding inclusion maps, are equivalences of triangulated categories.

Let us prove the equivalence on the left. By Lemma 5.9, it is enough to show that $\Xi: \mathcal{q}_{\mathrm{qis}}^{-1} \mathrm{~F}^{*}(\mathrm{~A}, \mathcal{T}, \mathcal{F}) \rightarrow \mathcal{8}_{\mathrm{qis}}^{-1} \mathrm{Ch}^{*}(\mathrm{~A}, \mathcal{T}, \mathcal{F})$ is an equivalence. We will prove this using the first case of Lemma 5.8.

Let $\left(E^{\bullet}, M^{\bullet}\right) \in \mathrm{Ch}^{*}(\mathrm{~A}, \mathcal{T}, \mathcal{F})$. Since the torsion theory is cotilting, we can find a complex $F^{\bullet}$ with terms all in $\mathcal{F}$, together with an epimorphic quasi-isomorphism $f: F^{\bullet} \rightarrow E^{\bullet}$. It follows from Lemma 7.5 that $\left(F^{\bullet}, f^{-1}\left(M^{\bullet}\right)\right)$ is in $\mathrm{F}^{*}(\mathrm{~A}, \mathcal{\mathcal { T }}, \mathcal{F})$. The quasi-isomorphism $\left(F^{\bullet}, f^{-1}\left(M^{\bullet}\right)\right) \rightarrow\left(E^{\bullet}, M^{\bullet}\right)$ guarantees that Lemma 5.8 applies. This proves that $\Xi$ is an equivalence.

The proof that $\delta_{\mathrm{qis}}^{-1} \mathrm{~F}^{*}(\mathrm{~A}) \rightarrow D^{*}(\mathrm{~A})$ is an equivalence is entirely similar.

\section{Iterating the construction for B}

The abelian category B comes with a torsion pair $\left(\mathcal{T}^{\prime}, \mathcal{F}^{\prime}\right)$, where $\mathcal{T}^{\prime}=\mathscr{F}[1]$ and $\mathscr{F}^{\prime}=\mathcal{T}$. We describe the abelian category $C$ obtained by applying the tilting procedure to this torsion pair.

8.1. Objects of C. It follows from the description of kernels and cokernels in Section 3.4 that an object in $\mathrm{C}$ is a diagram

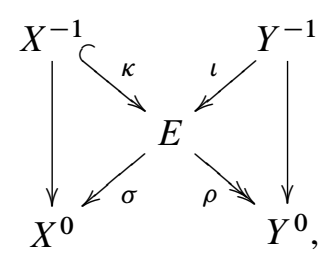

where $\kappa$ is a monomorphism and $\rho$ is an epimorphism. Equivalently, an object in C is a 4-tuple $\left[K_{1} \subseteq K_{2} \subseteq E \supseteq M\right]$ satisfying the following conditions: 
- $K_{2} \cap M \in \mathcal{F}$,

- $E /\left(K_{1}+M\right) \in \mathcal{T}$.

(Take $K_{1}=\kappa\left(X^{-1}\right), K_{2}=\operatorname{ker} \rho$, and $M=\iota\left(Y^{-1}\right)$.)

8.2. Morphisms of C. A strict morphism $\left[K_{1} \subseteq K_{2} \subseteq E \supseteq M\right] \rightarrow\left[K_{1}^{\prime} \subseteq K_{2}^{\prime} \subseteq\right.$ $\left.E^{\prime} \supseteq M^{\prime}\right]$ is a morphism $f: E \rightarrow E^{\prime}$ in A respecting the three subobjects. Let us denote the category of such 4-tuples and strict morphisms between them by $\mathrm{C}^{s t}$. Note that $\mathrm{C}^{s t}$ is naturally identified with a full subcategory of $\mathrm{Ch}^{s t}(\mathrm{~B})$. We denote $\delta_{\text {qis }} \cap \mathrm{C}^{s t}$ by the same notation $\delta_{\text {qis }}$; see Section 4 for notation. In other words, a morphism in $\mathrm{C}^{s t}$ is in $\oiint_{\text {qis }}$ if it induces an isomorphism $K_{1} / K_{2} \stackrel{\sim}{\longrightarrow} K_{1}^{\prime} / K_{2}^{\prime}$.

Proposition 8.1. The inclusion $i: \mathrm{C}^{\text {st }} \rightarrow \mathrm{C}$ induces an additive equivalence of abelian categories $8_{\mathrm{qis}}^{-1} \mathrm{C}^{\text {st }} \stackrel{\sim}{\rightarrow}$. Furthermore, the functor

$$
H: \mathrm{C}^{\mathrm{st}} \rightarrow \mathrm{A}, \quad\left[K_{1} \subseteq K_{2} \subseteq E \supseteq M\right] \mapsto K_{2} / K_{1},
$$

fits into the following commutative (up to a canonical natural transformation) diagram:

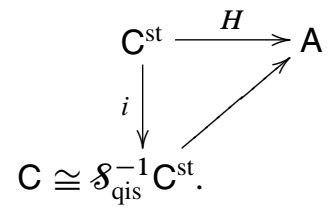

(Also, note that ${\delta_{\mathrm{qis}}^{-1}}^{-1} \mathrm{C}^{\mathrm{st}}$ is exactly the class of morphisms $\mathrm{C}^{\text {st }}$ that map to isomorphisms in $\mathrm{A}$ under $\mathrm{H}$.)

Proof. That $i: \mathrm{C}^{\text {st }} \rightarrow \mathrm{C}$ induces an equivalence of additive categories $\delta_{\mathrm{qis}}^{-1} \mathrm{C}^{\text {st }} \stackrel{\sim}{\longrightarrow} \mathrm{C}$ follows from Lemma 4.4. The existence of the commutative triangle is trivial.

The functor $\mathrm{C} \rightarrow \mathrm{A}$, which we will, by abuse of notation, denote by $H$, is known to be an equivalence of abelian categories whenever $(\mathcal{T}, \mathcal{F})$ is either tilting or cotilting. Let us give a quick proof of this.

Theorem 8.2 (Happel-Reiten-Smalø). Assume $(\mathcal{T}, \mathcal{F})$ is either tilting or cotilting. Then the functor $H: \mathrm{C} \rightarrow \mathrm{A}$ defined above is an additive equivalence of categories.

Proof. Assume $(\mathcal{T}, \mathcal{F})$ is tilting. We define the inverse functor $Q: \mathrm{A} \rightarrow \mathrm{C}$ as follows. For every $A \in \mathrm{A}$ choose a monomorphism $i_{A}: A \hookrightarrow T$ with $T \in \mathcal{T}$. The effect of $Q$ on objects is $Q(A):=[0 \subseteq A \subseteq T \supseteq 0]$. To define the effect of $Q$ on morphisms, let $f: A \rightarrow A^{\prime}$ be a morphism in A, and let $Q\left(A^{\prime}\right)=\left[0 \subseteq A^{\prime} \subseteq T^{\prime} \supseteq 0\right]$. Set 
$X=\left[0 \subseteq A \subseteq T \oplus T^{\prime} \supseteq 0\right]$, where $A \hookrightarrow T \oplus T^{\prime}$ is the map $\left(i_{A}, i_{A^{\prime}} \circ f\right)$. We define $Q(f)$ to be the roof

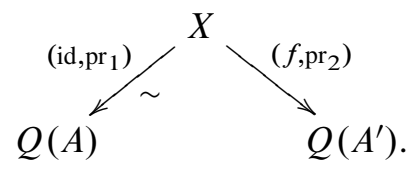

It is easy to check that $Q$ is a well-defined functor and that it is an inverse equivalence to $H$.

Proof in the cotilting case is similar. The functor $Q^{\prime}: \mathrm{A} \rightarrow \mathrm{C}$ sends an object $A \in \mathrm{A}$ to $[$ ker $p \subseteq F \subseteq F \supseteq F$ ], where $p: F \rightarrow A$ is a choice of an epimorphism with $F \in \mathscr{F}$.

\section{The DG structure}

The category $\mathrm{Ch}(\mathrm{B})$ is naturally a DG category, and so is $\mathrm{Ch}(\mathrm{A}, \mathcal{\mathcal { T }}, \mathcal{F})$, as we will see shortly. So one would like to strengthen Theorem 7.3 to a statement about DG categories. We will do that in this and the next section.

We will assume that $\mathrm{A}$ is a $K$-linear abelian category, where $K$ is a commutative ring.

9.1. The symmetric monoidal category $\operatorname{Dec}(K)$. Let $\operatorname{Dec}(K)$ denote the category of decorated complexes of $K$-modules. This is a symmetric monoidal category. Given two decorated complexes $\left(E^{\bullet}, M^{\bullet}\right)$ and $\left(F^{\bullet}, N^{\bullet}\right)$, their tensor product is the complex $E^{\bullet} \otimes F^{\bullet}$, decorated with the image of $\left(E^{\bullet} \otimes N^{\bullet}\right) \oplus\left(M^{\bullet} \otimes F^{\bullet}\right)$.

We discuss the inner homs in Section 9.2.

9.2. The $\operatorname{Dec}(K)$ enrichment of $\operatorname{Dec}(\mathbf{A})$. We will introduce a $\operatorname{Dec}(K)$ enrichment of $\operatorname{Dec}(A)$, which we denote by $\operatorname{Dec}(A)$. This is stronger than a DG structure.

Given two objects $\left(E^{\bullet}, M^{\bullet}\right)$ and $\left(F^{\bullet}, N^{\bullet}\right)$ in $\operatorname{Dec}(\mathrm{A})$, we define the $K$-complex

$$
\mathfrak{S o m}\left(\left(E^{\bullet}, M^{\bullet}\right),\left(F^{\bullet}, N^{\bullet}\right)\right)
$$

to be the subcomplex of the usual mapping complex $\mathfrak{5} o m\left(E^{\bullet}, F^{\bullet}\right)$ consisting of maps satisfying certain compatibility with respect to $M^{\bullet}$ and $N^{\bullet}$. More precisely, an element in the $k$-th term of the complex $\mathfrak{5} \operatorname{om}\left(\left(E^{\bullet}, M^{\bullet}\right),\left(F^{\bullet}, N^{\bullet}\right)\right)$ is a collection of morphisms $g_{n}: E^{n} \rightarrow F^{n+k}, n \in \mathbb{Z}$ such that the following conditions are satisfied:

(1) For every $n, g_{n}\left(M^{n}\right) \subseteq N^{n+k}$. 
(2) For every $n, \delta_{F} \circ g_{n}-(-1)^{k} g_{n+1} \circ \delta_{E}$ maps $M^{n}$ to $N^{n+k+1}$. Equivalently, the following diagram commutes:

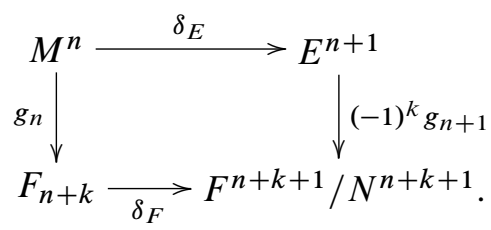

The differential $d$ on $\mathfrak{H} o m\left(\left(E^{\bullet}, M^{\bullet}\right),\left(F^{\bullet}, N^{\bullet}\right)\right)$ is defined as usual:

$$
\begin{aligned}
d: \operatorname{Sgom}\left(\left(E^{\bullet}, M^{\bullet}\right),\left(F^{\bullet}, N^{\bullet}\right)\right)^{k} & \rightarrow \operatorname{Som}\left(\left(E^{\bullet}, M^{\bullet}\right),\left(F^{\bullet}, N^{\bullet}\right)\right)^{k+1}, \\
\left\{g_{n}\right\}_{n \in \mathbb{Z}} & \mapsto\left\{\delta_{F} \circ g_{n}-(-1)^{k} g_{n+1} \circ \delta_{E}\right\}_{n \in \mathbb{Z}} .
\end{aligned}
$$

It is not hard to verify that the sequence $\left\{\delta_{F} \circ g_{n}-(-1)^{k} g_{n+1} \circ \delta_{E}\right\}_{n \in \mathbb{Z}}$ also satisfies (1) and (2).

There is a natural decoration

$$
\mathcal{M}\left(\left(E^{\bullet}, M^{\bullet}\right),\left(F^{\bullet}, N^{\bullet}\right)\right) \subseteq \mathfrak{S o m}\left(\left(E^{\bullet}, M^{\bullet}\right),\left(F^{\bullet}, N^{\bullet}\right)\right)
$$

on $\mathscr{5} \operatorname{om}\left(\left(E^{\bullet}, M^{\bullet}\right),\left(F^{\bullet}, N^{\bullet}\right)\right)$ whose $k^{\text {th }}$ term is, by definition, the $K$-submodule of $\mathfrak{5}$ om $\left(\left(E^{\bullet}, M^{\bullet}\right),\left(F^{\bullet}, N^{\bullet}\right)\right)^{k}$ consisting of those sequences $\left\{g_{n}\right\}_{n \in \mathbb{Z}}$ satisfying the following axiom:

- For every $n, g_{n}\left(M^{n}\right)=0$ and $g_{n}\left(E^{n}\right) \subseteq N^{n}$. Note that this condition implies (1) and (2).

9.3. The $\operatorname{Dec}(K)$ enrichment of $\operatorname{Ch}(A, \mathcal{T}, \mathcal{F})$. The category $\operatorname{Ch}(A, \mathcal{T}, \mathcal{F})$, being a full subcategory of $\operatorname{Dec}(\mathrm{A})$, inherits a $\operatorname{Dec}(K)$ enrichment, which we denote by $\mathfrak{E} \mathfrak{h}(\mathrm{A}, \mathcal{T}, \mathcal{F})$. This $\operatorname{Dec}(K)$ enrichment has a special feature that is described in the next proposition. We simplify the notation by denoting $\mathcal{M}\left(\left(E^{\bullet}, M^{\bullet}\right),\left(F^{\bullet}, N^{\bullet}\right)\right)$ by $\mathcal{M}$ and $\mathfrak{S} o m\left(\left(E^{\bullet}, M^{\bullet}\right),\left(F^{\bullet}, N^{\bullet}\right)\right)$ by $\mathfrak{S} o m$.

Proposition 9.1. For every $k$, $\mathcal{M}^{k} \cap d^{-1}\left(\mathcal{M}^{k+1}\right)=0$. That is, $H^{-1, k}($ Som, $\mathcal{M})=0$, for all $k$; see Section 5.2 for notation.

Proof. Let $\left\{g_{n}\right\}_{n \in \mathbb{Z}}$ be in $\mathcal{M}^{k} \cap d^{-1}\left(\mathcal{M}^{k+1}\right)$. Then the following are true for all $n$ :

(i) $g_{n}\left(M^{n}\right)=0$,

(ii) $g_{n}\left(E^{n}\right) \subseteq N^{n+k}$,

(iii) $\delta_{F} \circ g_{n}-(-1)^{k} g_{n+1} \circ \delta_{E}$ vanishes on $M^{n}$, and

(iv) $\delta_{F} \circ g_{n}-(-1)^{k} g_{n+1} \circ \delta_{E}$ maps $E^{n}$ to $N^{n+k+1}$. 
It follows from (i) and (iii), both with $n-1$, that $g_{n}$ vanishes on $\delta_{E}\left(M^{n-1}\right)$. This implies that $g_{n}$ factors through

$$
H^{0, n-1}\left(E^{\bullet}, M^{\bullet}\right)=E^{n} /\left(M^{n}+\delta_{E}\left(M^{n-1}\right) .\right.
$$

Also, it follows from (iv), with $n$, and (ii), with $n+1$, that $g_{n}\left(E^{n}\right) \subseteq \delta_{F}^{-1}\left(N^{n+k+1}\right)$. Therefore, $g_{n}$ factors through

$$
H^{-1, n+k}\left(F^{\bullet}, N^{\bullet}\right)=N^{n+k} \cap \delta_{F}^{-1}\left(N^{n+k+1}\right) .
$$

Put together, we see that $g_{n}$ factors through a map

$$
H^{0, n-1}\left(E^{\bullet}, M^{\bullet}\right) \rightarrow H^{-1, n+k}\left(F^{\bullet}, N^{\bullet}\right) .
$$

Since the left-hand side belongs to $\mathcal{T}$ and the right-hand side belongs to $\mathcal{F}$, this map has to be zero. Hence, $g_{n}=0$.

Corollary 9.2. The decorated complex $(5 \mathrm{Hom}, \mathcal{M})$ is naturally quasi-isomorphic to the complex $\mathscr{H}^{0}(5 \mathrm{Hom}, \mathcal{M})[-1]$ endowed with the zero decoration; see Section 5.2 for notation.

This corollary means that $\mathfrak{C} \mathfrak{h}(A, \mathcal{T}, \mathcal{F})$ should simply be thought of as a DG category, with the hom complexes being $\mathscr{H}^{0}(\mathfrak{H} o m, \mathcal{M})[-1]$.

Abuse of notation. We denote the DG category whose objects are the ones of $\mathrm{Ch}(\mathrm{A}, \mathcal{T}, \mathcal{F})$ and whose hom complexes are $\mathscr{H}^{0}(\mathfrak{H o m}, \mathcal{M})[-1]$ also by $\mathfrak{C} \mathfrak{h}(\mathrm{A}, \mathcal{T}, \mathcal{F})$.

9.4. DG structures on $\mathbf{C h}(\mathbf{B})$ and $\mathbf{C h}^{\text {st }}(\mathrm{B})$. Being the category of chain complexes in a $K$-linear abelian category, $\mathrm{Ch}(\mathrm{B})$ carries a natural $K$-linear DG structure, which we denote by $\mathfrak{E} \mathfrak{h}(\mathrm{B})$. The DG structure of $\mathfrak{E h}(\mathrm{B})$ induces a DG structure on $\mathrm{Ch}^{\text {st }}(\mathrm{B})$ as

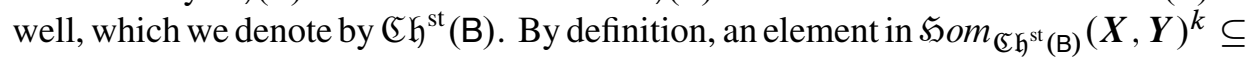
$\mathfrak{S}_{\mathfrak{H} m_{\mathfrak{C h}(\mathrm{B})}}(\boldsymbol{X}, \boldsymbol{Y})^{k}$ is a sequence of morphisms $h_{n}:{ }^{n} \mathbb{X} \rightarrow{ }^{n+k} Y, n \in \mathbb{Z}$, satisfying the following conditions:

- Every $h_{n}$ is strict;

- The dotted arrow in the following diagram can be filled:

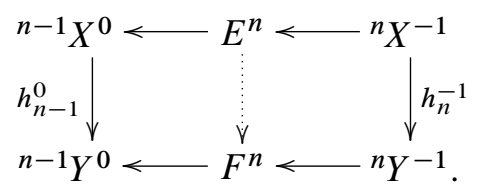

Here ${ }^{n} \mathbb{X}=\left[{ }^{n} X^{-1} \rightarrow{ }^{n} X^{0}\right],{ }^{n} Y=\left[{ }^{n} Y^{-1} \rightarrow{ }^{n} Y^{0}\right]$, and $E^{n}$ and $F^{n}$ are the extensions that define the morphisms $d:{ }^{n-1} \mathbb{X} \rightarrow{ }^{n} \mathbb{X}$ and $d:{ }^{n-1} Y \rightarrow{ }^{n} Y$.

That $\mathfrak{H}_{\mathfrak{H}} m_{\mathfrak{C h} \mathfrak{h}^{\text {st }}(\mathrm{B})}(\boldsymbol{X}, \boldsymbol{Y})$ is indeed a subcomplex of $\mathfrak{S} o m_{\mathfrak{C h}(\mathrm{B})}(\boldsymbol{X}, \boldsymbol{Y})$ is an easy exercise (it also follows from the proof of Proposition 10.1 in the next section). 
9.5. Remark: decorations of length $\boldsymbol{l}$. A decorated complex can be thought of as a complex each of whose terms is equipped with a length one filtration (but the differentials do not necessarily respect the filtrations). We can drop the requirement on the length of the filtration and consider complexes with length $l$ decorations, $0 \leq l \leq \infty$. It turns out that the category $\operatorname{Dec}^{l}(K)$ of chain complexes of $K$ modules with length $l$ decorations has a natural closed monoidal structure, and for every $K$-linear category A, the category $\operatorname{Dec}^{l}(\mathrm{~A})$ of chain complexes in A with length $l$ decorations is naturally enriched over $\operatorname{Dec}^{l}(K)$. The homological algebra of decorated complexes, as described in Section 5.5, carries over to $\operatorname{Dec}^{l}(K)$.

\section{The derived DG equivalence between $\mathfrak{C h}^{b}(B)$ and $\mathfrak{C h} \mathfrak{h}^{b}(A, \mathcal{T}, \mathcal{F})$}

The functor $G: \operatorname{Ch}(\mathrm{A}, \mathcal{T}, \mathcal{F}) \rightarrow \mathrm{Ch}(\mathrm{B})$ defined in the proof of Theorem 6.1 can be enriched to a DG functor $\mathbb{S}: \mathfrak{C h}(A, \mathcal{T}, \mathcal{F}) \rightarrow \mathfrak{C h}^{\text {st }}(\mathrm{B})$ in the obvious way; see the proof of Proposition 10.1 below. We will use this to construct a DG equivalence between $\mathfrak{E} \mathfrak{h}^{b}(\mathrm{~B})$ and $\mathfrak{C} \mathfrak{h}^{b}(\mathrm{~A}, \mathcal{T}, \mathcal{F})$ strengthening the derived equivalence of Theorem 7.3; see Theorem 10.9 below.

Proposition 10.1. The functor \& induces a DG equivalence

$$
\mathfrak{S}: \mathfrak{C h}(A, \mathcal{T}, \mathcal{F}) \rightarrow \mathfrak{C h}^{\text {st }}(\mathrm{B}) .
$$

Before proving the proposition, we prove a lemma.

Lemma 10.2. A strict morphism $\left(f^{-1}, f^{0}\right):\left[X^{-1} \stackrel{d_{X}}{\longrightarrow} X^{0}\right] \rightarrow\left[Y^{-1} \stackrel{d_{Y}}{\longrightarrow} Y^{0}\right]$ in $\mathrm{B}$ is the zero morphism if and only if there exists $s: X^{0} \rightarrow Y^{-1}$ such that $f^{0}=d_{Y} \circ s$ and $f^{-1}=s \circ d_{X}$, that is, $\left(f^{-1}, f^{0}\right)$ is null-homotopic in $\mathrm{Ch}(\mathrm{A})$.

Proof. Use the description of strict morphisms in Section 3.1.

Proof of Proposition 10.1. Let $\left(E^{\bullet}, M^{\bullet}\right)$ and $\left(F^{\bullet}, N^{\bullet}\right)$ be objects in $\mathfrak{C h}(\mathrm{A}, \mathcal{T}, \mathcal{F})$, and set $\boldsymbol{X}=G\left(E^{\bullet}, M^{\bullet}\right)$ and $\boldsymbol{Y}=G\left(F^{\bullet}, N^{\bullet}\right)$, as in the proof of Theorem 6.1 (p. 247).

Consider an element $\gamma$ in $\mathfrak{5 o m}\left(\left(E^{\bullet}, M^{\bullet}\right),\left(F^{\bullet}, N^{\bullet}\right)\right)^{k}$ given by the sequence $g_{n}: E^{n} \rightarrow F^{n+k}, n \in \mathbb{Z}$. By (1) of p. 252, each $g_{n}$ induces maps $\underline{g}_{n}: M^{n} \rightarrow N^{n+k}$ and $\bar{g}_{n}: E^{n} / M^{n} \rightarrow F^{n+k} / N^{n+k}$. Condition (2) guarantees that the map

$\circlearrowleft(\gamma)_{n}:=\left((-1)^{k} \underline{g}_{n}, \bar{g}_{n+1}\right):\left[M^{n} \rightarrow E^{n+1} / M^{n+1}\right] \rightarrow\left[N^{n+k} \rightarrow F^{n+k+1} / N^{n+k+1}\right]$

is a morphism in B. (Recall that, by definition, ${ }^{n} \mathbb{X}=\left[M^{n} \rightarrow E^{n+1} / M^{n+1}\right]$ and $n+k Y=\left[N^{n+k} \rightarrow F^{n+k+1} / N^{n+k+1}\right]$.) We define $\circlearrowleft(\gamma)$ to be the sequence $\circlearrowleft(\gamma)_{n}$, 
$n \in \mathbb{Z}$. It is easy to see that the map of $K$-modules

$$
\mathfrak{S} \operatorname{orm}\left(\left(E^{\bullet}, M^{\bullet}\right),\left(F^{\bullet}, N^{\bullet}\right)\right)^{k} \rightarrow \mathfrak{H} o m_{\mathbb{C h}^{\mathrm{st}}(\mathrm{B})}(\boldsymbol{X}, \boldsymbol{Y})^{k}, \quad \gamma \mapsto \circlearrowleft(\gamma),
$$

is surjective. We claim that its kernel is equal to

$$
\mathcal{M}\left(\left(E^{\bullet}, M^{\bullet}\right),\left(F^{\bullet}, N^{\bullet}\right)\right)^{k}+d \mathcal{M}\left(\left(E^{\bullet}, M^{\bullet}\right),\left(F^{\bullet}, N^{\bullet}\right)\right)^{k-1} .
$$

By Lemma 10.2, applied to $\circlearrowleft(\gamma)_{n}$, a sequence $g_{n}: E^{n} \rightarrow F^{n+k}, n \in \mathbb{Z}$, is in the kernel of the above map if and only if there is a sequence $s_{n}: E^{n} \rightarrow F^{n+k-1}$ such that for all $n$

(i) $s_{n}\left(M^{n}\right)=0$,

(ii) $s_{n}\left(E^{n}\right) \subseteq N^{n+k-1}$,

(iii) $g_{n}-(-1)^{k} s_{n+1} \circ \delta_{E}$ vanishes on $M^{n}$, and

(iv) $g_{n}-\delta_{F} \circ s_{n}$ maps $E^{n}$ to $N^{n+k}$.

We clearly have $\sigma:=\left\{s_{n}\right\}_{n \in \mathbb{Z}} \in \mathcal{M}\left(\left(E^{\bullet}, M^{\bullet}\right),\left(F^{\bullet}, N^{\bullet}\right)\right)^{k-1}$. It is also straightforward from conditions (i)-(iv) above that

$$
\gamma-d(\sigma)=\left\{g_{n}-\delta_{F} \circ s_{n}+(-1)^{k-1} s_{n+1} \circ \delta_{E}\right\}_{n \in \mathbb{Z}} \in \mathcal{M}\left(\left(E^{\bullet}, M^{\bullet}\right),\left(F^{\bullet}, N^{\bullet}\right)\right)^{k} .
$$

This proves our claim about the kernel.

Abbreviating $\mathfrak{5} \mathrm{om}\left(\left(E^{\bullet}, M^{\bullet}\right),\left(F^{\bullet}, N^{\bullet}\right)\right)$ and $\mathcal{M}\left(\left(E^{\bullet}, M^{\bullet}\right),\left(F^{\bullet}, N^{\bullet}\right)\right)$ to $\mathfrak{H o m}$

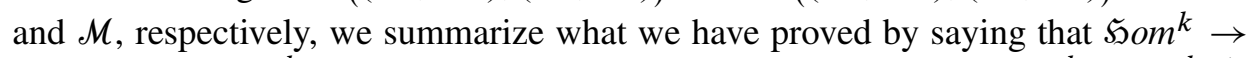
$\mathfrak{S} o m_{\mathfrak{C H}^{\mathrm{st}}(\mathrm{B})}(\boldsymbol{X}, \boldsymbol{Y})^{k}$ is a surjective map of $K$-modules whose kernel is $\mathcal{M}^{k}+d \mathcal{M}^{k-1}$. Therefore, we have an induced isomorphism

$$
\mathscr{H}^{0}(\mathfrak{S} \mathrm{om}, \mathcal{M})[-1]^{k}=\mathfrak{S} o m^{k} /\left(\mathcal{M}^{k}+d \mathcal{M}^{k-1}\right) \stackrel{\sim}{\longrightarrow} \mathfrak{H} o m_{\left(\mathfrak{C h}^{\mathrm{st}}(\mathrm{B})\right.}(\boldsymbol{X}, \boldsymbol{Y})^{k} .
$$

This is exactly what we wanted to prove.

Remark 10.3. The DG equivalence of the previous lemma is a DG equivalence in a strong sense: it induces isomorphisms on hom complexes.

It is easy to see that the functor Tot can also be enriched to a DG equivalence Tot: $\mathfrak{C H}^{\text {st }}(B) \rightarrow \mathfrak{C h}(A, \mathcal{T}, \mathcal{F})$, and that $\mathfrak{T}$ ot and $\mathbb{F}$ are inverse to each other.

Of course, we expect that applying $Z_{0}$ to the above enrichments give us back the old categories.

Proposition 10.4. We have equivalences

$$
\begin{aligned}
Z_{0} \mathfrak{C h}(\mathrm{B}) & \cong \mathrm{Ch}(\mathrm{B}), \\
Z_{0} \mathfrak{C h}^{s t}(\mathrm{~B}) & \cong \mathrm{Ch}^{\mathrm{st}}(\mathrm{B}), \\
Z_{0} \mathfrak{C h}(\mathrm{A}, \mathcal{T}, \mathcal{F}) & \cong \mathrm{Ch}(\mathrm{A}, \mathcal{T}, \mathcal{F}), \\
H_{0} \mathfrak{C h}(\mathrm{A}, \mathcal{T}, \mathcal{F}) & \cong \mathrm{K}(\mathrm{A}, \mathcal{T}, \mathcal{F}) .
\end{aligned}
$$

(See Section 5.5 for notation.) 
10.1. Semi-injective and semi-projective objects in $B$. Before we prove the derived equivalence of $\mathfrak{C} \mathfrak{h}(B)$ and $\mathfrak{C} \mathfrak{h}(A, \mathcal{T}, \mathcal{F})$ we need some definitions.

We say that an object $\mathbb{X}=\left[X^{-1} \rightarrow X^{0}\right]$ in $\mathrm{B}$ is semi-injective (respectively, semiprojective) if $X^{-1}$ an injective object in A (respectively, if $X^{0}$ a projective object in A). Note that these notions are not invariant under isomorphism.

Proposition 10.5. Let $\boldsymbol{X}$ and $\boldsymbol{Y}$ be complexes in $\mathrm{Ch}(\mathrm{B})$. Assume either $\boldsymbol{X}$ is a complex of semi-projective objects, or $\boldsymbol{Y}$ is a complex of semi-injective objects. Then

$$
\mathfrak{S}_{\mathfrak{H} o m \mathfrak{C h}^{\mathrm{st}}}(\boldsymbol{X}, \boldsymbol{Y}) \hookrightarrow \mathfrak{S}_{\mathfrak{H} o m_{\mathfrak{C h}}}(\boldsymbol{X}, \boldsymbol{Y})
$$

is an isomorphism. (We do not need any boundedness conditions on $\boldsymbol{X}$ or $\boldsymbol{Y}$ ).

Proof. This follows from Lemma 3.2.

Lemma 10.6. If $\mathrm{A}$ has enough injectives, then for every object $\mathbb{X}$ in $\mathrm{B}$ there exists a semi-injective object $\mathbb{I}$ and a strict isomorphism $\mathbb{X} \rightarrow \mathbb{I}$. If $\mathrm{A}$ has enough projectives, then for every object $\mathbb{X}$ in $\mathrm{B}$ there exists a semi-projective object $\mathbb{P}$ and a strict isomorphism $\mathbb{P} \rightarrow \mathbb{X}$.

Proof. Assume that A has enough injectives. Take a monomorphism $X^{-1} \hookrightarrow I$ into an injective $I$ and set $\mathbb{I}=\left[I \rightarrow X^{0} \oplus^{X^{-1}} I\right]$. Similarly, if A has enough projectives, take an epimorphism $P \rightarrow X^{0}$ from a projective $P$ and put $\mathbb{P}=\left[P \oplus_{X^{0}} X^{-1} \rightarrow P\right]$.

Corollary 10.7. Let $\boldsymbol{X}$ be a complex in $\mathrm{B}$. If $\mathrm{A}$ has enough injectives, then there is a complex $\boldsymbol{I}$ of semi-injective objects in $\mathrm{B}$ and a strict isomorphism $\boldsymbol{X} \rightarrow \boldsymbol{I}$. If $\mathrm{A}$ has enough projectives, then there is a complex $\mathbf{P}$ of semi-projective objects in $\mathrm{B}$ and a strict isomorphism $\boldsymbol{P} \rightarrow \boldsymbol{X}$. (No boundedness conditions needed.)

10.2. Derived equivalence of $\mathfrak{C h}^{b}(B)$ and $\mathfrak{C h}^{b}(A, \mathcal{T}, \mathcal{F})$. In this section we will need $A$ to have either enough injectives or enough projectives. Let us say that $A$ has enough projectives. For $\boldsymbol{X}, \boldsymbol{Y} \in \mathfrak{C h}^{\text {st }}(\mathrm{B})$ we define

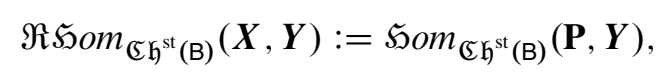

where $\boldsymbol{P} \rightarrow \boldsymbol{X}$ is a semi-projective resolution as in Corollary 10.7. If $\boldsymbol{P}^{\prime}$ is another semi-projective resolution for $\boldsymbol{X}$, it follows from Lemma 3.2 that there is a canonical strict isomorphism $\boldsymbol{P}^{\prime} \stackrel{\sim}{\longrightarrow} \boldsymbol{P}$ over $\boldsymbol{X}$, with a strict inverse $\boldsymbol{P} \stackrel{\sim}{\longrightarrow} \mathbf{P}^{\prime}$. Therefore, $\Re \mathfrak{R S O}_{\mathfrak{C h}^{\mathrm{st}}(\mathrm{B})}(\boldsymbol{X}, \boldsymbol{Y})$ is well defined up to a canonical isomorphism.

Proposition 10.5 implies that there is an isomorphism of $K$-complexes

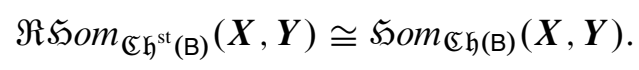


In other words, the inclusion $\mathfrak{E} \mathfrak{h}^{\text {st }}(\mathrm{B}) \hookrightarrow \mathfrak{C} \mathfrak{h}(\mathrm{B})$ is a "derived" equivalence; here "derived" refers to the localizing class $\delta_{\text {sis }}$ and not $\delta_{\text {qis }}$. By Proposition 10.1, we find that $\mathfrak{G}: \mathfrak{C h}(A, \mathcal{T}, \mathcal{F}) \rightarrow \mathfrak{C} \mathfrak{h}(B)$ is a "derived" equivalence. Similar discussion is valid in the case where $A$ has enough injectives. We summarize this in the following proposition.

Proposition 10.8. Assume that $\mathrm{A}$ has either enough injectives or enough projectives. Then we have a natural "derived" equivalence

$$
\mathfrak{C h}(\mathrm{B}) \cong \mathfrak{C} \mathfrak{h}(\mathrm{A}, \mathcal{T}, \mathcal{F})
$$

of DG categories. (Here "derived" refers to the localizing class $\wp_{\text {sis.) }}$.

From this we deduce the DG version of Theorem 7.3.

Theorem 10.9. Assume that A has either enough injectives or enough projectives. Assume further that B has enough injectives (respectively, enough projectives). Let $*=+, b$ (respectively, $*=-, b)$. Then we have a derived equivalence

$$
(\mathfrak{C h})^{*}(B) \cong \mathfrak{C h} \mathfrak{H}^{*}(A, \mathcal{T}, \mathcal{F})
$$

of DG categories. (Here "derived" refers to either of the two localizing class $\delta_{\mathrm{sis}}$ or $\oiint_{\text {qis. }}$.

Some explanation about the meaning of this theorem is perhaps helpful. First of all, the real interesting case of the theorem is when the localizing class is $\delta_{\text {qis }}$; that is what the word "derived" is usually associated with. We know that given $\boldsymbol{X}$ and $\boldsymbol{Y}$ in $\mathfrak{C h}^{*}(\mathrm{~B})$, the hom-complex $\mathfrak{5} \mathrm{wm}_{\mathfrak{C h}(\mathrm{B})}(\boldsymbol{X}, \boldsymbol{Y})$ is not well behaved with respect to quasi-isomorphisms. That is why, as in the case of derived categories, we are more interested in the derived hom-complexes, namely, the ones obtained by first replacing $\boldsymbol{X}$ and $\boldsymbol{Y}$ by an appropriate projective or injective resolution, and then taking $\mathfrak{H} \mathrm{om}$. What the above theorem is saying is that the functors between $\mathfrak{C} \mathfrak{h}^{*}(\mathrm{~B})$ and $\mathfrak{C h}^{*}(\mathrm{~A}, \mathcal{T}, \mathcal{F})$ do not necessarily induce quasi-isomorphisms on the usual homcomplexes $\mathfrak{H} o m_{(\mathfrak{C h}(\mathrm{B})}(\boldsymbol{X}, \boldsymbol{Y})$, but they do induce quasi-isomorphisms on the derived hom-complexes.

\section{References}

[Be] A. A. Beilinson, The derived category of coherent sheaves on $\mathbf{P}^{n}$. Selecta Math. Soviet. 3 (1984), 233-237. Zbl 0545.14012 MR 863137

[BeGePa] I. N. Bernstein, I. M. Gel'fand, and V. A. Ponomarev, Coxeter functors and Gabriel's theorem. Uspekhi Mat. Nauk 28 (2) (1973), 19-33; English transl. Russian Math. Surveys 28 (2) (1973), 17-32. Zbl 0279.08001 MR 0393065 
[Bo] A. I. Bondal, Representations of associative algebras and coherent sheaves. Izv. Akad. Nauk SSSR Ser. Mat. 53 (1989), 25-44; English transl. Math. USSR-Izv. 34 (1990), 23-42. Zbl 0692.18002 MR 992977

$[\mathrm{BrBu}] \quad \mathrm{S}$. Brenner and M. C. R. Butler, Generalizations of the Bernstein-Gel'fandPonomarev reflection functors. In Representation theory, II (Proc. Second Internat. Conf., Carleton Univ., Ottawa, Ont., 1979), Lecture Notes in Math. 832, Springer, Berlin 1980, 103-169. Zbl 0446.16031 MR 0607151

[Br1] T. Bridgeland, T-structures on some local Calabi-Yau varieties. J. Algebra 289 (2005), 453-483. Zbl 1069.14044 MR 2142382

[Br2] T. Bridgeland, Stability conditions on triangulated categories. Ann. of Math. (2) 166 (2007), 317-345. Zbl 1137.18008 MR 2373143

[GeMa] S. I. Gelfand and Yu. I. Manin, Methods of homological algebra. Springer-Verlag, Berlin 1996. Zbl 0855.18001 MR 1438306

[HaReSm] D. Happel, I. Reiten, and S. O. Smalø, Tilting in abelian categories and quasitilted algebras. Mem. Amer. Math. Soc. 120 (1996), no. 575. Zbl 0849.16011 MR 1327209

[No] B. Noohi, On weak maps between 2-groups. Preprint 2005. arXiv:math/0506313v3

[Po1] A. Polishchuk, Classification of holomorphic vector bundles on noncommutative two-tori. Doc. Math. 9 (2004), 163-181. Zbl 1048.32012 MR 2054986

[Po2] A. Polishchuk, Holomorphic bundles on 2-dimensional noncommutative toric orbifolds. In Noncommutative geometry and number theory, Aspects Math. E37, Vieweg, Wiesbaden 2006, 341-359. Zbl 1103.14002 MR 2327312

[PoSch] A. Polishchuk and A. Schwarz, Categories of holomorphic vector bundles on noncommutative two-tori. Comm. Math. Phys. 236 (2003), 135-159. Zbl 1033.58009 MR 1977884

[vdB] M. Van den Bergh, Abstract blowing down. Proc. Amer. Math. Soc. 128 (2000), 375-381. Zbl 0929.14004 MR 1628424

[We] C. A. Weibel, An introduction to homological algebra. Cambridge Stud. Adv. Math. 38, Cambridge University Press, Cambridge 1994. Zbl 0797.18001 MR 1269324

Received March 4, 2008; revised July 20, 2008

B. Noohi, Department of Mathematics, Florida State University, Tallahassee,

Florida 32306-4510, U.S.A.

E-mail: behrang@alum.mit.edu 